University of Windsor

Scholarship at UWindsor

$1-2014$

\title{
Incomplete interval fuzzy preference relations and their applications
}

\author{
Yejun Xu \\ Hohai University \\ Kevin Li \\ University of Windsor \\ Huimin Wang \\ Hohai University
}

Follow this and additional works at: https://scholar.uwindsor.ca/odettepub

Part of the Business Commons

\section{Recommended Citation}

Xu, Yejun; Li, Kevin; and Wang, Huimin. (2014). Incomplete interval fuzzy preference relations and their applications. Computers \& Industrial Engineering, 67, 93-103.

https://scholar.uwindsor.ca/odettepub/97

This Article is brought to you for free and open access by the Odette School of Business at Scholarship at UWindsor. It has been accepted for inclusion in Odette School of Business Publications by an authorized administrator of Scholarship at UWindsor. For more information, please contact scholarship@uwindsor.ca. 


\title{
Incomplete interval fuzzy preference relations and their applications
}

\begin{abstract}
This paper investigates incomplete interval fuzzy preference relations. An additive consistency property of fuzzy preference relations proposed by Herrera-Viedma et al. (2004) is first extended to a more general case. This property is then generalized to interval fuzzy preference relations (IFPRs) based on additive transitivity. Subsequently, we examine how to characterize additive consistent IFPR. Using these new characterizations, we propose a method to construct an additive consistent IFPR from an acceptable incomplete IFPR comprising $n-1$ preference data and an estimation algorithm for an acceptable incomplete IFPR with more known elements. Numerical examples are provided to illustrate the effectiveness and practicality of the proposed solution procedure.
\end{abstract}

Keywords: Incomplete interval fuzzy preference relation; Additive consistent; Group decision making.

\section{Introduction}

A fuzzy preference relation satisfying additive reciprocity is one of the most common preference relations for expressing a decision maker's (DM's) preference over alternatives. In a decision making process, the DM often needs to compare a set of $n$ decision alternatives $x_{i}$ $(i=1,2, \ldots, n)$, thereby constructing a fuzzy preference relation (Herrera-Viedma et al., 2004; Kacprzyk, 1986; Orlovsky, 1978; Tanino, 1984; Wang and Fan, 2007; Xu et al., 2009; Xu et al., 2013c; Xu, 2005). However, the DM may have vague knowledge about the preference degrees of one alternative over another and cannot estimate his/her preference with an exact numerical value, but with an interval number. In this case, the DM constructs an interval preference relation.

Saaty and Vargas (1987) first presented interval judgments as a way to model subjective uncertainty. Afterwards, different methods are proposed to generate weights from interval comparison matrices, such as linear programing (LP) (Arbel, 1989; Kress, 1991), lexicographic goal programming (LGP) (Islam et al., 1997; Wang, 2006), fuzzy preference programming (FPP) (Mikhailov, 2002; Mikhailov, 2004), two-stage logarithmic goal programming (TLGP) (Wang et al., 2005), eigenvector method (EM) (Wang and Chin, 2006), Lambda-Max method (Csutora and Buckley, 2001), goal programming method (GPM) (Wang and Elhag, 2007), etc. 
For IFPRs, Xu (2004b) defined the concept of a compatibility degree between two IFPRs, and showed the compatibility relationship between individual IFPRs and collective IFPR. Herrera et al.(2005) developed an aggregation process for combining IFPRs with other types of information such as numerical preference relation and linguistic preference relation. Jiang (2007) gave an index to measure the similarity degree between two IFPRs, and employed an error-propagation principle to determine a priority vector for the aggregated IFPRs. Recently, Xu and Chen (2008b) established some linear programming models for deriving priority weights from various IFPRs. Wang and Li (2012) developed goal-programming-based models for deriving interval weights from IFPRs for both individual and group decision-making situations.

The aforesaid research focused on preference relations with complete information. A complete preference relation of order $n$ necessitates the completion of all $n(n-1) / 2$ judgments in its entire top (lower) triangular portion where the lower (top) triangular part is implied by additive reciprocity. Sometimes, however, a DM may develop a preference relation with incomplete information due to a variety of reasons such as time pressure, lack of knowledge, and the DM's limited expertise related with the problem domain (Chiclana et al., 2008; Lee et al., 2007; Xu and Da, 2008; Xu and Da, 2009; Xu et al., 2010; Xu et al., 2013a; Xu et al., 2013b; Xu, 2004a; Xu, 2005; Xu and Chen, 2008a); In addition, when the number of the alternatives, $n$, is large, it may be impractical to require the DM to perform all the $n(n-1) / 2$ required comparisons for a complete pairwise comparison matrix (Fedrizzi and Silvio, 2007); Moreover, it is sometimes convenient or necessary to skip some direct comparison between alternatives even if the total number of alternatives is small (Fedrizzi and Silvio, 2007); In some other cases, a DM is unable to express any kind of preference between two or more options due to the expert's insufficient knowledge of the problem or inability in discriminating the degree to which one option is preferred to another (Alonso et al., 2004; Alonso et al., 2008; Herrera-Viedma et al., 2007a; Herrera-Viedma et al., 2007b; Xu, 2012).

A critical concern for incomplete fuzzy preference relations is to estimate the missing values. Herrera-Viedma et al. (2007a) proposed an iterative procedure to estimate the missing information in an expert's incomplete fuzzy preference relation. The procedure is guided by the additive consistency property and uses only the preference values provided by the expert. Fedrizzi and Giove (2007) put forward a new method for calculating missing elements in an incomplete fuzzy preference relation by maximizing global consistency. Later, Chiclana et al. (2009) pointed out that the two methods are very similar in calculating missing values. Chiclana et al. (2008) 
presented a new estimation method based on the U-consistency criterion for incomplete fuzzy preference relations. Alonso et al. (2008) presented a procedure to estimate missing preference values for incomplete fuzzy, multiplicative, interval-valued, and linguistic preference relations. Liu et al. (2012b) developed a method to calculate missing values by minimizing the squared error of an incomplete fuzzy preference relation and its priority weight vector. Xu (2012) devised an approach to extending each incomplete multiplicative preference relation to a complete one by exploiting multiplicative transitivity properties. Xia et al. (2012) furnished an algorithm to estimate missing values for an incomplete linguistic preference relation based on multiplicative consistency. Recently, research has been extended to interval-valued preference relations. For instance, Alonso et al. (2008) put forward a procedure to estimate missing information for the incomplete interval preference relations. Genç et al. (2010) examined consistency, missing value(s) and derivation of priority vectors from IFPRs based on multiplicative transitivity. Liu et al. (2012a) proposed a new method to obtain priority weights from incomplete interval multiplicative preference relations. However, limited research has been devoted to incomplete IFPRs. As such, it is necessary to pay attention to this issue.

Another important issue is the consistency of the judgment information provided by DMs (Chiclana et al., 2002; Herrera-Viedma et al., 2004). It is obvious that consistent information is more relevant and important than the information containing contradictions. Consistency is associated with certain transitivity properties. Different properties have been suggested to model transitivity of fuzzy preference relations. One of these properties is the "additive transitivity", which, as shown in by Herrera-Viedma et al. (2004), can be seen as a parallel concept to Saaty's consistency property for multiplicative reciprocal preference relations.

The aim of this paper is to propose methods for constructing additive consistent IFPRs based on acceptable incomplete IFPRs. We first extend an additive consistency property proposed by Herrera-Viedma et al. (2004) for fuzzy preference relations to a more general case. This property is then extended to IFPRs based on additive transitivity. After further characterizing additive consistent IFPRs, we develop two algorithms for estimating missing elements from acceptable incomplete IFPRs. A procedure is then laid out for handling GDM problems with acceptable incomplete IFPRs.

The rest of this paper is organized as follows. Section 2 reviews some properties of fuzzy preference relations. Section 3 first introduces the concepts of interval multiplicative reciprocal 
preference relations and IFPRs as well as their transformation function. The property of additive consistent fuzzy preference relations in Section 2 is then extended to IFPRs, followed by further characterizations of additive consistent IFPRs. Section 4 presents two approaches to construct additive consistent IFPRs based on acceptable incomplete IFPRs. A case study is furnished in Section 5 to illustrate how to apply our algorithms. We conclude the paper in Section 6.

\section{Additive consistent fuzzy preference relations}

Let $X=\left\{x_{1}, x_{2}, \ldots, x_{n}\right\}(n \geq 2)$ be a finite set of alternatives, where $x_{i}$ denotes the $i^{\text {th }}$ alternative. In multiple attribute decision making problems, a DM needs to rank alternatives $x_{1}, x_{2}, \ldots, x_{n}$ from the best to the worst according to preference information. A brief description of multiplicative and fuzzy preference relations is given below.

\subsection{Multiplicative preference relations}

A multiplicative preference relation is a positive preference relation $A \subset X \times X, A=\left(a_{i j}\right)_{n \times n}$, where $a_{i j}$ denotes the DM's relative preference of alternative $x_{i}$ over $x_{j}$. The measurement of $a_{i j}$ is described by a ratio scale and in particular, as shown by Saaty $(1980), a_{i j} \in\{1 / 9,1 / 8, \ldots, 1,2, \ldots$, $9\}: a_{i j}=1$ denotes the DM's indifference between $x_{i}$ and $x_{j}, a_{i j}=9\left(\right.$ or $\left.a_{j i}=1 / 9\right)$ denotes that $x_{i}$ is absolutely preferred to $x_{j}$, and $a_{i j} \in\{2,3, \ldots, 8\}$ denotes intermediate preference evaluations. This relation is multiplicative reciprocal, i.e., $a_{i j} a_{j i}=1, \forall i, j \in\{1,2, \ldots, n\}$ and in particular, $a_{i i}=1$, $\forall i \in\{1,2, \ldots, n\}$. Its consistency is defined by Saaty (1980) as follows.

Definition 1. Let $A=\left(a_{i j}\right)_{n \times n}$ be a multiplicative preference relation, then $A$ is called consistent (Saaty, 1980), if $a_{i j}=a_{i k} a_{k j}$, for all $i, j, k$.

\subsection{Fuzzy preference relations}

A fuzzy preference relation $R$ is described as follows: $R \subset X \times X, R=\left(r_{i j}\right)_{n \times n}$, with membership function $u_{R}: X \times X \rightarrow[0,1]$, where $u_{R}\left(x_{i}, x_{j}\right)=r_{i j}$ denotes the preference degree of alternative $x_{i}$ over $x_{j}$ (Kacprzyk, 1986; Tanino, 1984): $r_{i j}=0.5$ denotes indifference between $x_{i}$ 
and $x_{j}, r_{i j}=1$, denotes that $x_{i}$ is definitely preferred to $x_{j}$, and $0.5<r_{i j}<1 \quad\left(\right.$ or $0<r_{j i}<0.5$ ) denotes that $x_{i}$ is preferred to $x_{j}$ to a varying degree.

Definition 2. Let $R=\left(r_{i j}\right)_{n \times n}$ be a preference relation, then $R$ is called a fuzzy preference relation if

$$
r_{i j} \in[0,1], r_{i j}+r_{j i}=1, r_{i i}=0.5 \text {, for all } i, j \in N
$$

Definition 3. Let $R=\left(r_{i j}\right)_{n \times n}$ be a fuzzy preference relation, then $R$ is additive consistent if the following additive transitivity (Tanino, 1984) is satisfied:

$$
r_{i j}=r_{i k}-r_{j k}+0.5, \text { for all } i, j, k=1,2, \ldots, n
$$

\subsection{Characterizing additive consistency of fuzzy preference relations}

Herrera-Viedma et al. (2004) studied the transformation between multiplicative preference relations with values in the interval scale [1/9,9] (Alonso et al., 2004) and fuzzy preference relations with values in $[0,1]$ and furnished the following propositions.

Proposition 1 (Herrera-Viedma et al., 2004). Consider a set of alternatives $X=\left\{x_{1}, x_{2}, \ldots, x_{n}\right\}$, associated with a multiplicative reciprocal preference relation $A=\left(a_{i j}\right)_{n \times n}$ with $a_{i j} \in[1 / 9,9]$. Then, a corresponding fuzzy preference relation, $R=\left(r_{i j}\right)_{n \times n}$, with $r_{i j} \in[0,1]$, associated with $A$ is given as follows:

$$
r_{i j}=g\left(a_{i j}\right)=\frac{1}{2}\left(1+\log _{9} a_{i j}\right)
$$

Proposition 2 (Herrera-Viedma et al., 2004). For a fuzzy preference relation $R=\left(r_{i j}\right)_{n \times n}$, the following statements are equivalent:

(a) $r_{i j}+r_{j k}+r_{k i}=\frac{3}{2}, \forall i, j, k$;

(b) $r_{i j}+r_{j k}+r_{k i}=\frac{3}{2}, i<j<k$.

Proposition 3 (Herrera-Viedma et al., 2004). For a fuzzy preference relation $R=\left(r_{i j}\right)_{n \times n}$, the following statements are equivalent: 
(a) $r_{i j}+r_{j k}+r_{k i}=\frac{3}{2}, i<j<k$;

(b) $r_{i(i+1)}+r_{(i+1)(i+2)}+\ldots+r_{(j-1) j}+r_{j i}=\frac{j-i+1}{2}, i<j$.

\subsection{A new characterization of additive consistency}

Herrera-Viedma et al. (2004) showed that Proposition 3 can be used to construct an additive consistent fuzzy preference relation from a set of $n-1$ values $\left\{r_{12}, r_{23}, \ldots, r_{n-1 n}\right\}$. The aforesaid propositions were also used by Wang and Chen $(2007 ; 2008)$. In the following, a more general result is provided.

Proposition 4. For a fuzzy preference relation $R=\left(r_{i j}\right)_{n \times n}$, the following statements are equivalent:

(a) $r_{i j}+r_{j k}+r_{k i}=\frac{3}{2}, \forall i, j, k \in N$;

(b) $r_{i j_{1}}+r_{j_{1} j_{2}}+\ldots+r_{j_{t-1} j_{t}}+r_{j_{t} i}=\frac{t+1}{2}, \forall i, j_{l} \in N, l=1,2, \ldots, t$.

Proof. $(a) \Rightarrow(b)$ Mathematical induction is employed to prove this part of the proposition. It is obviously true for $t=1$ as it is reduced to the additive reciprocity property in Definition 2. Next, if the hypothesis is true for $t=n$,

$$
r_{i j_{1}}+r_{j_{1} j_{2}}+\ldots+r_{j_{n-2} j_{n-1}}+r_{j_{n-1} j_{n}}+r_{j_{n} i}=\frac{n+1}{2}
$$

we shall prove that it is also true for $t=n+1$

$$
\begin{aligned}
r_{i j_{1}}+r_{j_{1} j_{2}}+\ldots+r_{j_{n-1} j_{n}}+r_{j_{n} j_{n+1}}+r_{j_{n+1} i}=\left(r_{i j_{1}}+r_{j_{1} j_{2}}+\ldots+r_{j_{n-2} j_{n-1}}+r_{j_{n-1} j_{n}}\right)+r_{j_{n} j_{n+1}}+r_{j_{n+1} i} \\
=\frac{n+1}{2}-r_{j_{n} i}+r_{j_{n} j_{n+1}}+r_{j_{n+1} i} \\
=\frac{n-1}{2}+r_{i j_{n}}+r_{j_{n} j_{n+1}}+r_{j_{n+1} i}=\frac{n-1}{2}+\frac{3}{2}=\frac{n+2}{2}
\end{aligned}
$$

So the result is established.

$(b) \Rightarrow(a)$.

$$
\begin{aligned}
r_{i j}+r_{j k} & +r_{k i}=1-r_{j i}+1-r_{k j}+r_{k i} \\
& =2+\left(r_{i j_{1}}+\ldots+r_{j_{t-1}}-\frac{t+1}{2}\right)+\left(r_{j j_{1}}+\ldots+r_{j_{t-1} k}-\frac{t+1}{2}\right)+r_{k i}
\end{aligned}
$$




$$
\begin{aligned}
& =1-t+\left(r_{i j_{1}}+\ldots+r_{j_{t-1} j}+r_{j j_{1}}+\ldots+r_{j_{t-1} k}+r_{k i}\right) \\
& =1-t+\frac{2 t+1}{2}=\frac{3}{2}
\end{aligned}
$$

This completes the proof.

Furthermore, in the proof process, it is obvious that the differences of $j_{2}-j_{1}, j_{3}-j_{2}, \ldots, j_{t}-j_{t-1}$ are not necessarily equal to 1 . As a matter of fact, the differences do not have to be identical.

Proposition 4 differs from Proposition 3 in that any sequence of values $r_{i j_{1}}, r_{j_{1} j_{2}}, \ldots, r_{j_{t-1} j_{t}}, r_{j_{t} i}$ (for example $\left.r_{31}, r_{15}, r_{57}, r_{73}\right)$ will work for Proposition 4. But Proposition 3 requires preference values to follow a consecutive order such as $r_{12}, r_{23}, \ldots, r_{n-1 n}, r_{n 1}$. Therefore, Proposition 4 includes Proposition 3 as a special case.

\section{Interval fuzzy preference relations and their characterizations}

In the following, we shall first introduce some operational laws for interval numbers (Hayes, 2003; Moore, 1966). We then present a relationship between an interval multiplicative preference relation and an IFPR. The characterizations of additive consistent fuzzy preference relations in Section 2 are subsequently extended to IFPRs, followed by other useful results.

Let $\bar{a}_{1}=\left[a_{1}^{-}, a_{1}^{+}\right], \bar{a}_{2}=\left[a_{2}^{-}, a_{2}^{+}\right], \bar{a}=\left[a^{-}, a^{+}\right]$be three positive interval numbers, then

(a) $\bar{a}_{1} \oplus \bar{a}_{2}=\left[a_{1}^{-}, a_{1}^{+}\right] \oplus\left[a_{2}^{-}, a_{2}^{+}\right]=\left[a_{1}^{-}+a_{2}^{-}, a_{1}^{+}+a_{2}^{+}\right]$;

(b) $\bar{a}_{1} \ominus \bar{a}_{2}=\left[a_{1}^{-}, a_{1}^{+}\right] \ominus\left[a_{2}^{-}, a_{2}^{+}\right]=\left[a_{1}^{-}-a_{2}^{+}, a_{1}^{+}-a_{2}^{-}\right]$;

(c) $\bar{a}_{1} \otimes \bar{a}_{2}=\left[a_{1}^{-}, a_{1}^{+}\right] \otimes\left[a_{2}^{-}, a_{2}^{+}\right]=\left[a_{1}^{-} \times a_{2}^{-}, a_{1}^{+} \times a_{2}^{+}\right]$;

(d) $\log _{n}(\bar{a})=\left[\log _{n} a^{-}, \log _{n} a^{+}\right]$;

(e) $\bar{a}^{-1}=\left[1 / a^{+}, 1 / a^{-}\right]$.

Definition 4. An interval preference relation $\bar{A}=\left(\bar{a}_{i j}\right)$ is multiplicative reciprocal if and only if $\bar{a}_{j i}=\bar{a}_{i j}^{-1}$.

Definition $5(\mathrm{Xu}, 2010)$. If a positive interval multiplicative reciprocal preference relation $\bar{A}=\left(\bar{a}_{i j}\right)$ satisfies:

$$
\bar{a}_{i j}=\bar{a}_{i k} \otimes \bar{a}_{k j}, \text { for all } i, k, j=1,2, \ldots, n, \text { and } i \leq k \leq j
$$


then $\bar{A}=\left(\bar{a}_{i j}\right)$ is multiplicative consistent.

Note that if an interval multiplicative reciprocal preference relation is multiplicative consistent, for all $i, k, j \in\{1,2, \ldots, n\}$, as pointed out by $\mathrm{Xu}$ (2010), it is necessary to require $i \leq k \leq j$, otherwise, $\bar{A}=\left(\bar{a}_{i j}\right)$ would be reduced to a crisp number judgment matrix (Saaty's multiplicative reciprocal preference relation). For more detail, readers are referred to (Xu, 2010). The implication is that it is only necessary to verify Eq.(9) for the upper (or lower) triangular portion of the preference relation when multiplicative consistency is checked.

Definition $6(\mathrm{Xu}, 2004 \mathrm{~b})$. Let $\bar{R}=\left(\overline{r_{i j}}\right)$ be an interval valued preference relation, if

$$
\begin{aligned}
& \bar{r}_{i j}=\left[r_{i j}^{-}, r_{i j}^{+}\right], \bar{r}_{i j}=\left[r_{j i}^{-}, r_{j i}^{+}\right], r_{i j}^{-}+r_{j i}^{+}=r_{i j}^{+}+r_{j i}^{-}=1, \\
& r_{i j}^{+} \geq r_{i j}^{-} \geq 0, \text { for all } i, j=1,2, \ldots, n
\end{aligned}
$$

then $\bar{R}$ is called an interval fuzzy preference relation (IFPR).

Definition 7. Let $\bar{R}=\left(\overline{r_{i j}}\right)$ be an IFPR, if

$$
\bar{r}_{i j} \oplus \bar{r}_{j k}=\bar{r}_{i k} \oplus[0.5,0.5], \forall i<j<k
$$

then $\bar{R}$ is called an additive consistent IFPR.

Proposition 5. For a set of alternatives $X=\left\{x_{1}, x_{2}, \ldots, x_{n}\right\}$, and its associated interval multiplicative reciprocal preference relation $\bar{A}=\left(\bar{a}_{i j}\right)$ with $\bar{a}_{j i}=\bar{a}_{i j}^{-1}$, a corresponding IFPR, $\bar{R}=\left(\overline{r_{i j}}\right)$, associated with $\bar{A}$ is given as

$$
\bar{r}_{i j}=g\left(\bar{a}_{i j}\right)=\frac{1}{2}\left([1,1] \oplus \log _{9} \bar{a}_{i j}\right)
$$

such that

(a) $r_{i j}^{-}+r_{j i}^{+}=1, \forall i, j \in N$;

(b) $r_{i j}^{+}+r_{j i}^{-}=1 . \forall i, j \in N$.

Proof. As $\bar{A}=\left(\bar{a}_{i j}\right)$ is an interval multiplicative reciprocal preference relation, by Definition 4 , $\bar{a}_{j i}=\bar{a}_{i j}^{-1}$, that is

$$
\left[a_{j i}^{-}, a_{j i}^{+}\right]=\left[\frac{1}{a_{i j}^{+}}, \frac{1}{a_{i j}^{-}}\right]
$$

Thus 


$$
\begin{aligned}
& a_{j i}^{-}=\frac{1}{a_{i j}^{+}}, a_{j i}^{+}=\frac{1}{a_{i j}^{-}} . \\
& a_{j i}^{-} a_{i j}^{+}=1, a_{j i}^{+} a_{i j}^{-}=1
\end{aligned}
$$

By Proposition 1 and Eq.(11) and the operational law (Eq.(7)) for interval numbers, we have

$$
\bar{r}_{i j}=\frac{1}{2}\left(1 \oplus \log _{9} \bar{a}_{i j}\right)=\frac{1}{2}\left([1,1] \oplus \log _{9}\left[a_{i j}^{-}, a_{i j}^{+}\right]\right)
$$

Thus

$$
\begin{aligned}
& r_{i j}^{-}=\frac{1}{2}\left(1+\log _{9} a_{i j}^{-}\right) \\
& r_{i j}^{+}=\frac{1}{2}\left(1+\log _{9} a_{i j}^{+}\right)
\end{aligned}
$$

Similarly,

$$
\begin{aligned}
& r_{j i}^{-}=\frac{1}{2}\left(1+\log _{9} a_{j i}^{-}\right) \\
& r_{j i}^{+}=\frac{1}{2}\left(1+\log _{9} a_{j i}^{+}\right)
\end{aligned}
$$

Therefore,

$$
\begin{aligned}
& r_{i j}^{-}+r_{j i}^{+}=\frac{1}{2}\left(1+\log _{9} a_{i j}^{-}\right)+\frac{1}{2}\left(1+\log _{9} a_{j i}^{+}\right)=1+\log _{9} a_{i j}^{-} a_{j i}^{+}=1, \\
& r_{i j}^{+}+r_{j i}^{-}=\frac{1}{2}\left(1+\log _{9} a_{i j}^{+}\right)+\frac{1}{2}\left(1+\log _{9} a_{j i}^{-}\right)=1+\log _{9} a_{i j}^{+} a_{j i}^{-}=1, \forall i, j \in\{1,2, \ldots, n\}
\end{aligned}
$$

The proof is thus completed.

Next, we examine the relationship between multiplicative consistency of an interval multiplicative reciprocal preference relation and additive consistency of its converted IFPR as per Eq.(11).

Proposition 6. If an interval multiplicative reciprocal preference relation $\bar{A}=\left(\bar{a}_{i j}\right)$ is multiplicative consistent, then its corresponding IFPR $\bar{R}=\left(\bar{r}_{i j}\right)$ is additive consistent, and

(a) $r_{i j}^{-}+r_{j k}^{-}+r_{k i}^{+}=\frac{3}{2}, \forall i<j<k$;

(b) $r_{i j}^{+}+r_{j k}^{+}+r_{k i}^{-}=\frac{3}{2}, \forall i<j<k$; 
(c) $r_{i(i+1)}^{-}+r_{(i+1)(i+2)}^{-}+\ldots+r_{(j-1) j}^{-}+r_{j i}^{+}=\frac{j-i+1}{2}, \forall i<j$;

(d) $r_{i(i+1)}^{+}+r_{(i+1)(i+2)}^{+}+\ldots+r_{(j-1) j}^{+}+r_{j i}^{-}=\frac{j-i+1}{2}, \forall i<j$;

(e) $r_{i j_{1}}^{-}+r_{j_{1} j_{2}}^{-}+\ldots+r_{j_{t-1} j_{t}}^{-}+r_{j_{t} i}^{+}=\frac{t+1}{2}, \forall i<j_{1}<j_{2}<\ldots<j_{t}$;

(f) $r_{i_{1}}^{+}+r_{j_{1} j_{2}}^{+}+\ldots+r_{j_{t-1} j_{t}}^{+}+r_{j_{i}}^{-}=\frac{t+1}{2}, \forall i<j_{1}<j_{2}<\ldots<j_{t}$.

Proof. Since $\bar{A}=\left(\bar{a}_{i j}\right)$ is multiplicative consistent, by Definition $5, \bar{a}_{i j} \otimes \bar{a}_{j k}=\bar{a}_{i k}$, for $\forall i<j<k$.

Taking a logarithm operation (Eq.(7)) on both sides yields

$$
\log _{9} \bar{a}_{i j} \oplus \log _{9} \bar{a}_{j k}=\log _{9} \bar{a}_{i k}, \forall i<j<k
$$

Thus

$$
\frac{1}{2}\left([1,1] \oplus \log _{9} \bar{a}_{i j}\right) \oplus \frac{1}{2}\left([1,1] \oplus \log _{9} \bar{a}_{j k}\right)=\frac{1}{2}\left([1,1] \oplus \log _{9} \bar{a}_{i k}\right) \oplus[0.5,0.5]
$$

By Eq.(11), we have

$$
\begin{aligned}
& \bar{r}_{i j} \oplus \bar{r}_{j k}=\bar{r}_{i k} \oplus[0.5,0.5] \\
& {\left[r_{i j}^{-}, r_{i j}^{+}\right] \oplus\left[r_{j k}^{-}, r_{j k}^{+}\right]=\left[r_{i k}^{-}, r_{i k}^{+}\right] \oplus[0.5,0.5]} \\
& r_{i j}^{-}+r_{j k}^{-}=r_{i k}^{-}+0.5, r_{i j}^{+}+r_{j k}^{+}=r_{i k}^{+}+0.5
\end{aligned}
$$

By Proposition 5, we have

$$
r_{i j}^{-}+r_{j k}^{-}+r_{k i}^{+}=\frac{3}{2}, r_{i j}^{+}+r_{j k}^{+}+r_{k i}^{-}=\frac{3}{2}, \forall i<j<k
$$

Thus the expressions (a) and (b) are established.

Let $i<j$, and $k=j-i$. The expression (c) can be rewritten as follows:

$$
r_{i(i+1)}^{-}+r_{(i+1)(i+2)}^{-}+\ldots+r_{(j-1) j}^{-}+r_{j i}^{+}=\frac{k+1}{2}, \forall i<j
$$

Mathematical induction is used to prove this part. It is clearly true for $k=1$. Next if the hypothesis is true for $k=n$

$$
r_{i(i+1)}^{-}+r_{(i+1)(i+2)}^{-}+\ldots+r_{(i+n-1)(i+n)}^{-}+r_{(i+n) i}^{+}=\frac{n+1}{2}
$$

then for $k=n+1$ :

$$
r_{i(i+1)}^{-}+r_{(i+1)(i+2)}^{-}+\ldots+r_{(i+n-1)(i+n)}^{-}+r_{(i+n)(i+n+1)}^{-}+r_{(i+n+1) i}^{+}
$$




$$
\begin{aligned}
& =\left(r_{i(i+1)}^{-}+r_{(i+1)(i+2)}^{-}+\ldots+r_{(i+n-1)(i+n)}^{-}\right)+r_{(i+n)(i+n+1)}^{-}+r_{(i+n+1) i}^{+} \\
& =\left(\frac{n+1}{2}-r_{(i+n) i}^{+}\right)+r_{(i+n)(i+n+1)}^{-}+r_{(i+n+1) i}^{+} \\
& =\left(\frac{n+1}{2}+r_{i(i+n)}^{-}-1\right)+r_{(i+n)(i+n+1)}^{-}+r_{(i+n+1) i}^{+} \\
& =\frac{n-1}{2}+\frac{3}{2}=\frac{n+2}{2}
\end{aligned}
$$

thus the expression (c) is confirmed and (d) can be analogously asserted. Similar to Proposition 4, (e)-(f) can be verified.

Proposition 7. An IFPR $\bar{R}=\left(\bar{r}_{i j}\right)$ is additive consistent if and only if (1) $r_{i j}^{-}-r_{i k}^{-}=r_{l j}^{-}-r_{l k}^{-}$; $r_{i j}^{+}-r_{i k}^{+}=r_{l j}^{+}-r_{l k}^{+}, \forall i<j<k, l=1,2, \ldots, n$.

Proof. If an IFPR $\bar{R}=\left(\overline{r_{i j}}\right)$ is additive consistent by Definition 7 , then

$$
\begin{aligned}
& \bar{r}_{i j} \oplus \bar{r}_{j k}=\bar{r}_{i k} \oplus[0.5,0.5], \quad \forall i<j<k \\
& r_{i j}^{-}+r_{j k}^{-}=r_{i k}^{-}+\frac{1}{2}, r_{i j}^{+}+r_{j k}^{+}=r_{i k}^{+}+\frac{1}{2}, \forall i<j<k \\
& r_{i j}^{-}-r_{i k}^{-}=\frac{1}{2}-r_{j k}^{-}, r_{i j}^{+}-r_{i k}^{+}=\frac{1}{2}-r_{j k}^{+}, \forall i<j<k
\end{aligned}
$$

Similarly,

$$
\begin{aligned}
& \bar{r}_{l j} \oplus \bar{r}_{j k}=\bar{r}_{l k} \oplus[0.5,0.5], \forall l<j<k \\
& r_{l j}^{-}+r_{j k}^{-}=r_{l k}^{-}+\frac{1}{2}, r_{l j}^{+}+r_{l k}^{+}=r_{l k}^{+}+\frac{1}{2}, \forall l<j<k \\
& r_{l j}^{-}-r_{l k}^{-}=\frac{1}{2}-r_{j k}^{-}, r_{l j}^{+}-r_{l k}^{+}=\frac{1}{2}-r_{l k}^{+}, \forall l<j<k
\end{aligned}
$$

thus

$$
r_{i j}^{-}-r_{i k}^{-}=r_{l j}^{-}-r_{l k}^{-}, r_{i j}^{+}-r_{i k}^{+}=r_{l j}^{+}-r_{l k}^{+}, \forall i, l<j<k .
$$

On the contrary,

$$
\text { If } r_{i j}^{-}-r_{i k}^{-}=r_{l j}^{-}-r_{l k}^{-}, r_{i j}^{+}-r_{i k}^{+}=r_{l j}^{+}-r_{l k}^{+}, \forall i<j<k
$$

Let $l=j$, since $r_{j j}^{-}=r_{j j}^{+}=0.5$, then

$$
r_{i j}^{-}-r_{i k}^{-}=0.5-r_{j k}^{-}, r_{i j}^{+}-r_{i k}^{+}=0.5-r_{j k}^{+}, \forall i<j<k
$$


That is

$$
\begin{aligned}
& r_{i j}^{-}+r_{j k}^{-}=0.5+r_{i k}^{-}, r_{i j}^{+}+r_{j k}^{+}=0.5+r_{i k}^{+}, \forall i<j<k \\
& \bar{r}_{i j} \oplus \bar{r}_{j k}=\bar{r}_{i k} \oplus[0.5,0.5], \forall i<j<k
\end{aligned}
$$

This completes the proof.

Proposition 7 reveals an important property of an additive consistent IFPR. For upper (or lower) triangular interval values, the difference of the lower bounds between any two corresponding columns should be a constant for all rows. The same is true for the upper bounds of the interval preference values. Proposition 6 and Proposition 7 will play an important role in devising our algorithms to construct complete IFPRs based on an incomplete relation.

Note that, if the original multiplicative reciprocal preference values in $\bar{A}=\left(\bar{a}_{i j}\right)$ are not assessed on the $\{1 / 9,1 / 8, \ldots, 8,9\}$ scale, we may have obtained a matrix $\bar{R}$ with entries not in the interval $[0,1]$, but in an interval $[-c, 1+c]$, where $c>0,-c$ indicates the minimum value in matrix $\bar{R}, 1+c$ gives the maximum value of matrix $R$. In this case, the obtained values have to be converted using a transformation function that preserves reciprocity and additive consistency, i.e., $f:[-c, 1+c] \rightarrow[0,1]$, verifying

(a) $f(-c)=0$;

(b) $f(1+c)=1$;

(c) $f\left(x^{-}\right)+f\left(x^{+}\right)=1, \forall x \in[-c, 1+c]$;

(d) If $x^{-}+y^{-}+z^{+}=\frac{3}{2}, f\left(x^{-}\right)+f\left(y^{-}\right)+f\left(z^{+}\right)=\frac{3}{2}, \forall x^{-}, y^{-}, z^{+} \in[-c, 1+c]$;

(e) If $x^{+}+y^{+}+z^{-}=\frac{3}{2}, f\left(x^{+}\right)+f\left(y^{+}\right)+f\left(z^{-}\right)=\frac{3}{2}, \forall x^{+}, y^{+}, z^{-} \in[-c, 1+c]$

A linear function satisfying (a) and (b) takes the form

$$
\begin{aligned}
& f\left(x^{-}\right)=\varphi x^{-}+\beta, \varphi, \beta \in R ; \\
& f\left(x^{+}\right)=\varphi x^{+}+\beta, \varphi, \beta \in R ;
\end{aligned}
$$

If $\varphi=\frac{1}{1+2 c}, \beta=\frac{c}{1+2 c}$, then

$$
f\left(x^{-}\right)=\frac{1}{1+2 c} x^{-}+\frac{c}{1+2 c}=\frac{x^{-}+c}{1+2 c}
$$




$$
f\left(x^{+}\right)=\frac{1}{1+2 c} x^{+}+\frac{c}{1+2 c}=\frac{x^{+}+c}{1+2 c}
$$

(c) can be easily verified as

$$
f\left(x^{-}\right)+f\left(x^{+}\right)=\frac{x^{-}+c}{1+2 c}+\frac{x^{+}+c}{1+2 c}=\frac{\left(x^{-}+x^{+}\right)+2 c}{1+2 c}=1
$$

If $x^{-}+y^{-}+z^{+}=\frac{3}{2}, x^{+}+y^{+}+z^{-}=\frac{3}{2}$

$$
\begin{aligned}
f\left(x^{-}\right)+f\left(y^{-}\right)+f\left(z^{+}\right) & =\frac{x^{-}+c}{1+2 c}+\frac{y^{-}+c}{1+2 c}+\frac{z^{+}+c}{1+2 c} \\
& =\frac{\left(x^{-}+y^{-}+z^{+}\right)+3 c}{1+2 c}=\frac{3 / 2+3 c}{1+2 c}=\frac{3}{2} \\
f\left(x^{+}\right)+f\left(y^{+}\right)+f\left(z^{-}\right) & =\frac{x^{+}+c}{1+2 c}+\frac{y^{+}+c}{1+2 c}+\frac{z^{-}+c}{1+2 c} \\
& =\frac{\left(x^{+}+y^{+}+z^{-}\right)+3 c}{1+2 c}=\frac{3 / 2+3 c}{1+2 c}=\frac{3}{2}
\end{aligned}
$$

(d) and (e) are also confirmed.

Proposition 8. Let $\bar{R}^{(1)}, \bar{R}^{(2)}, \ldots, \bar{R}^{(m)}$ be $m$ IFPRs, then their weighted average

$$
\bar{R}=\lambda_{1} \bar{R}^{(1)} \oplus \lambda_{2} \bar{R}^{(2)} \oplus \ldots \oplus \lambda_{m} \bar{R}^{(m)}, \lambda_{l} \in[0,1], \sum_{l=1}^{m} \lambda_{l}=1
$$

is also an IFPR such that

$$
r_{i j}^{-}+r_{j i}^{+}=1, r_{i j}^{+}+r_{j i}^{-}=1, \forall i, j \in\{1,2, \ldots, n\}
$$

Proof. Since $\bar{R}^{(1)}, \bar{R}^{(2)}, \ldots, \bar{R}^{(m)}$ are IFPRs, it follows that

$$
r_{i j}^{(l)-}+r_{j i}^{(l)+}=1, r_{i j}^{(l)+}+r_{j i}^{(l)-}=1, \forall i, j \in\{1,2, \ldots, n\}, l=1,2, \ldots, m
$$

Then by Eq.(20), we have

$$
\begin{aligned}
& r_{i j}^{-}=\lambda_{1} r_{i j}^{(1)-}+\lambda_{2} r_{i j}^{(2)-}+\ldots+\lambda_{m} r_{i j}^{(m)-}, r_{i j}^{+}=\lambda_{1} r_{i j}^{(1)+}+\lambda_{2} r_{i j}^{(2)+}+\ldots+\lambda_{m} r_{i j}^{(m)+}, \forall i, j \in\{1,2, \ldots, n\} \\
& r_{j i}^{-}=\lambda_{1} r_{j i}^{(1)-}+\lambda_{2} r_{j i}^{(2)-}+\ldots+\lambda_{m} r_{j i}^{(m)-}, r_{j i}^{+}=\lambda_{1} r_{j i}^{(1)+}+\lambda_{2} r_{j i}^{(2)+}+\ldots+\lambda_{m} r_{j i}^{(m)+}, \forall i, j \in\{1,2, \ldots, n\} \\
& r_{i j}^{-}+r_{j i}^{+}=\lambda_{1}\left(r_{i j}^{(1)-}+r_{j i}^{(1)+}\right)+\lambda_{2}\left(r_{i j}^{(2)-}+r_{j i}^{(2)+}\right)+\ldots+\lambda_{m}\left(r_{i j}^{(m)-}+r_{j i}^{(m)+}\right) \\
& \quad=\lambda_{1}+\lambda_{2}+\ldots+\lambda_{m}=1 \\
& r_{i j}^{+}+r_{j i}^{-}=\lambda_{1}\left(r_{i j}^{(1)+}+r_{j i}^{(1)-}\right)+\lambda_{2}\left(r_{i j}^{(2)+}+r_{j i}^{(2)-}\right)+\ldots+\lambda_{m}\left(r_{i j}^{(m)+}+r_{j i}^{(m)-}\right) \\
& =\lambda_{1}+\lambda_{2}+\ldots+\lambda_{m}=1
\end{aligned}
$$


The proof is thus completed.

Proposition 9. Let $\bar{R}^{(1)}, \bar{R}^{(2)}, \ldots, \bar{R}^{(m)}$ be $m$ additive consistent IFPRs, then their weighted average

$$
\bar{R}=\lambda_{1} \bar{R}^{(1)} \oplus \lambda_{2} \bar{R}^{(2)} \oplus \ldots \oplus \lambda_{m} \bar{R}^{(m)}, \lambda_{l} \in[0,1], \sum_{l=1}^{m} \lambda_{l}=1
$$

is also an additive consistent IFPR.

Proof. Since $\bar{R}^{(1)}, \bar{R}^{(2)}, \ldots, \bar{R}^{(m)}$ are additive consistent IFPRs by Definition 7 .

$$
\bar{r}_{i j}^{(l)} \oplus \bar{r}_{j k}^{(l)}=\bar{r}_{i k}^{(l)} \oplus[0.5,0.5], \forall i<j<k
$$

then by Eq.(21), we have

$$
\begin{aligned}
& \bar{r}_{i j}=\lambda_{1} \bar{r}_{i j}^{(1)} \oplus \lambda_{2} \bar{r}_{i j}^{(2)} \oplus \ldots \oplus \lambda_{m} \bar{r}_{i j}^{(m)} \\
& \bar{r}_{j k}=\lambda_{1} \bar{r}_{j k}^{(1)} \oplus \lambda_{2} \bar{r}_{j k}^{(2)} \oplus \ldots \oplus \lambda_{m} \bar{r}_{j k}^{(m)} \\
& \bar{r}_{i k}=\lambda_{1} \bar{r}_{i k}^{(1)} \oplus \lambda_{2} \bar{r}_{i k}^{(2)} \oplus \ldots \oplus \lambda_{m} \bar{r}_{i k}^{(m)} \\
& \bar{r}_{i j} \oplus \bar{r}_{j k}=\lambda_{1}\left(\bar{r}_{i j}^{(1)} \oplus \bar{r}_{j k}^{(1)}\right) \oplus \lambda_{2}\left(\bar{r}_{i j}^{(2)} \oplus \bar{r}_{j k}^{(2)}\right) \oplus \ldots \oplus \lambda_{m}\left(\bar{r}_{i j}^{(m)} \oplus \bar{r}_{j k}^{(m)}\right) \\
& =\lambda_{1}\left(\bar{r}_{i k}^{(1)} \oplus[0.5,0.5]\right) \oplus \lambda_{2}\left(\bar{r}_{i k}^{(2)} \oplus[0.5,0.5]\right) \oplus \ldots \oplus \lambda_{m}\left(\bar{r}_{i k}^{(m)} \oplus[0.5,0.5]\right) \\
& =\lambda_{1} \bar{r}_{i k}^{(1)} \oplus \lambda_{2} \bar{r}_{i k}^{(2)} \oplus \ldots \oplus \lambda_{m} \bar{r}_{i k}^{(m)} \oplus\left[\left(\lambda_{1}+\lambda_{2}+\ldots+\lambda_{m}\right) / 2,\left(\lambda_{1}+\lambda_{2}+\ldots+\lambda_{m}\right) / 2\right] \\
& =\bar{r}_{i k} \oplus[0.5,0.5]
\end{aligned}
$$

This completes the proof.

\section{Procedures for constructing complete IFPRs based on acceptable incomplete IFPRs}

\subsection{Incomplete interval fuzzy preference relations}

A complete $n \times n$ preference relation requires $n(n-1) / 2$ judgments in its entire top (or lower) triangular portion. Sometimes, however, a DM may furnish a preference relation with incomplete information due to a variety of reasons. Next, we first present basic concepts of incomplete IFPRs, then develop two procedures for estimating missing values.

Definition 8. Let $\bar{R}=\left(\bar{r}_{i j}\right)$ be an $n \times n$ preference relation, if at least one element in the upper (or lower) triangular part is not given by the DM, denoted by an unknown variable " $x$ ", and remaining interval values provided by the DM satisfy

$$
\bar{r}_{i j}=[1,1] \ominus \bar{r}_{j i}, \bar{r}_{i i}=[0.5,0.5], \text { for all } \bar{r}_{i j} \in \Omega
$$

then $\bar{R}$ is called an incomplete IFPR, where $\Omega$ is the set of all the known elements in $\bar{R}$. 
Definition 9. The elements $\bar{r}_{i j}, \bar{r}_{k l}$ of $\bar{R}$ are called adjacent if $\{i, j\} \cap\{k, l\} \neq \varnothing$.

For a missing element $\bar{r}_{i j}$, it can be determined indirectly if there exist a sequence of adjacent known elements $\bar{r}_{i_{1}}, \bar{r}_{j_{1} j_{2}}, \ldots, \bar{r}_{j_{t}}$.

Definition 10. Let $\bar{R}=\left(\bar{r}_{i j}\right)$ be an incomplete IFPR. If all missing elements of $\bar{R}$ can be determined by the known elements, then $\bar{R}$ is called an acceptable incomplete IFPR. Otherwise, $\bar{R}$ is an unacceptable incomplete IFPR.

Next, we extend the necessary condition (Herrera-Viedma et al., 2007b; Xu and Da, 2008) of acceptable incomplete fuzzy preference relations to the case of incomplete IFPRs.

Proposition 10. Let $\bar{R}=\left(\overline{r_{i j}}\right)$ be an incomplete IFPR. If $\bar{R}$ is an acceptable incomplete IFPR, then there exists at least one known non-diagonal element in each row or each column of $\bar{R}$, i.e. there exist at least ( $n-1)$ judgments provided by the DM.

Definition 11. Let $\bar{R}=\left(\bar{r}_{i j}\right)$ be an incomplete IFPR, if the known elements satisfy

$$
\begin{aligned}
& r_{i_{1}}^{-}+r_{j_{1} j_{2}}^{-}+\ldots+r_{j_{t-1} j_{t}}^{-}+r_{j_{i} i}^{+}=\frac{t+1}{2}, \text { for } \forall i<j_{1}<j_{2}<\ldots<j_{t} \\
& r_{i_{1}}^{+}+r_{j_{1} j_{2}}^{+}+\ldots+r_{j_{t-1} j_{t}}^{+}+r_{j_{i} i}^{-}=\frac{t+1}{2}, \text { for } \forall i<j_{1}<j_{2}<\ldots<j_{t},
\end{aligned}
$$

then $\bar{R}$ is called an additive consistent incomplete IFPR.

\subsection{An estimation procedure for acceptable incomplete IFPRs with the fewest number of judgments}

Next, by exploiting Proposition 5 and Proposition 6 or Proposition 7, a simple and practical method is developed for constructing a complete additive consistent IFPR based on an acceptable incomplete IFPR with the fewest number of judgment data (i.e., $n-1$ preference values):

\section{Algorithm I}

Step 1. For a decision problem, let $X=\left\{x_{1}, x_{2}, \ldots, x_{n}\right\}$ be a discrete set of alternatives. The DM conducts pairwise comparisons among the alternatives and furnished his/her assessment as an acceptable incomplete IFPR $\bar{R}=\left(\bar{r}_{i j}\right)_{n \times n}$ (if the DM provides his/her evaluation as an acceptable incomplete interval multiplicative reciprocal preference relation $\bar{A}=\left(\bar{a}_{i j}\right)_{n \times n}$, then $\bar{A}=\left(\bar{a}_{i j}\right)_{n \times n}$ can 
be converted to a corresponding incomplete IFPR $\bar{R}=\left(\bar{r}_{i j}\right)_{n \times n}$ by Propositions 5), with only $n-1$ judgments.

Step 2. Utilizing Proposition 6 or Proposition 7 to determine all unknown elements in $\bar{R}$, and yield an additive consistent interval preference relation $\dot{\bar{R}}=\left(\dot{\bar{r}}_{i j}\right)_{n \times n}$. If this preference relation contains any values falling outside the unit interval $[0,1]$, but within the interval $[-c, 1+c]$, then a transformation function $f(x)=\frac{x+c}{1+2 c}$ can be applied to preserve the reciprocity and additive transitivity, resulting in an additive consistent IFPR.

Step 3. End.

Example 1. Assume that a decision problem involves evaluating seven faculties $x_{i}(i=1,2, \ldots, 7)$ at a university. The DM assesses these seven faculties (alternatives) by pairwise comparison and provides his/her judgment as follows:

$$
\bar{r}_{31}=[0.4,0.7], \bar{r}_{32}=[0.1,0.3], \bar{r}_{34}=[0.3,0.4], \bar{r}_{35}=[0.4,0.7], \bar{r}_{36}=[0.3,0.8], \bar{r}_{37}=[0.4,0.9] .
$$

Step 1. By Definition 8 and the aforesaid information provided by the DM, one obtains the following acceptable incomplete IFPR, where " $x$ " denotes the unknown judgment.

$$
\bar{R}=\left[\begin{array}{ccccccc}
{[0.5,0.5]} & x & x & x & x & x & x \\
x & {[0.5,0.5]} & x & x & x & x & x \\
{[0.4,0.7]} & {[0.1,0.3]} & {[0.5,0.5]} & {[0.3,0.4]} & {[0.4,0.7]} & {[0.3,0.8]} & {[0.4,0.9]} \\
x & x & x & {[0.5,0.5]} & x & x & x \\
x & x & x & x & {[0.5,0.5]} & x & x \\
x & x & x & x & x & {[0.5,0.5]} & x \\
x & x & x & x & x & x & {[0.5,0.5]}
\end{array}\right]
$$

Step 2. Utilize Proposition 6 and Proposition 7 to determine all missing elements in $\bar{R}$ as follows:

$$
\begin{aligned}
& r_{13}^{-}=1-r_{31}^{+}=0.3, r_{13}^{+}=1-r_{31}^{-}=0.6, r_{23}^{-}=1-r_{32}^{+}=0.7, r_{23}^{+}=1-r_{32}^{-}=0.9, \\
& r_{12}^{-}=\frac{3}{2}-r_{23}^{-}-r_{31}^{+}=0.1, r_{12}^{+}=\frac{3}{2}-r_{23}^{+}-r_{31}^{-}=0.2, r_{21}^{-}=1-r_{12}^{+}=0.8, r_{21}^{+}=1-r_{12}^{-}=0.9, \\
& r_{23}^{-}-r_{33}^{-}=r_{24}^{-}-r_{34}^{-} \Rightarrow r_{24}^{-}=r_{23}^{-}-r_{33}^{-}+r_{34}^{-}=0.5, r_{24}^{+}=r_{23}^{+}-r_{33}^{+}+r_{34}^{+}=0.8, \\
& r_{25}^{-}=r_{23}^{-}-r_{33}^{-}+r_{35}^{-}=0.6, r_{25}^{+}=r_{23}^{+}-r_{33}^{+}+r_{35}^{+}=1.1, \\
& r_{26}^{-}=r_{23}^{-}-r_{33}^{-}+r_{36}^{-}=0.5, r_{26}^{+}=r_{23}^{+}-r_{33}^{+}+r_{36}^{+}=1.2,
\end{aligned}
$$




$$
\begin{aligned}
& r_{27}^{-}=r_{23}^{-}-r_{33}^{-}+r_{37}^{-}=0.6, r_{27}^{+}=r_{23}^{+}-r_{33}^{+}+r_{37}^{+}=1.3 \text {, } \\
& r_{14}^{-}=r_{13}^{-}-r_{33}^{-}+r_{34}^{-}=0.1, r_{14}^{+}=r_{13}^{+}-r_{33}^{+}+r_{34}^{+}=0.5 \text {, } \\
& r_{15}^{-}=r_{13}^{-}-r_{33}^{-}+r_{35}^{-}=0.2, r_{15}^{+}=r_{13}^{+}-r_{33}^{+}+r_{35}^{+}=0.8 \text {, } \\
& r_{16}^{-}=r_{13}^{-}-r_{33}^{-}+r_{36}^{-}=0.1, r_{16}^{+}=r_{13}^{+}-r_{33}^{+}+r_{36}^{+}=0.9 \text {, } \\
& r_{17}^{-}=r_{13}^{-}-r_{33}^{-}+r_{37}^{-}=0.2, r_{17}^{+}=r_{13}^{+}-r_{33}^{+}+r_{37}^{+}=1 \text {, } \\
& r_{45}^{-}=r_{35}^{-}-r_{34}^{-}+r_{44}^{-}=0.6, r_{45}^{+}=r_{35}^{+}-r_{34}^{+}+r_{44}^{+}=0.8 \text {, } \\
& r_{46}^{-}=r_{36}^{-}-r_{34}^{-}+r_{44}^{-}=0.5, r_{46}^{+}=r_{36}^{+}-r_{34}^{+}+r_{44}^{+}=0.9 \text {, } \\
& r_{47}^{-}=r_{37}^{-}-r_{34}^{-}+r_{44}^{-}=0.6, r_{47}^{+}=r_{37}^{+}-r_{34}^{+}+r_{44}^{+}=1 \text {, } \\
& r_{56}^{-}=r_{36}^{-}-r_{35}^{-}+r_{55}^{-}=0.4, r_{56}^{+}=r_{36}^{+}-r_{35}^{+}+r_{55}^{+}=0.6 \\
& r_{57}^{-}=r_{37}^{-}-r_{35}^{-}+r_{55}^{-}=0.5, r_{57}^{+}=r_{37}^{+}-r_{35}^{+}+r_{55}^{+}=0.7 \text {, } \\
& r_{67}^{-}=r_{37}^{-}-r_{36}^{-}+r_{66}^{-}=0.6, r_{67}^{+}=r_{37}^{+}-r_{36}^{+}+r_{66}^{+}=0.6 \\
& r_{41}^{-}=1-r_{14}^{+}=0.5, r_{41}^{+}=1-r_{14}^{-}=0.9, r_{42}^{-}=1-r_{24}^{+}=0.2, r_{42}^{+}=1-r_{24}^{-}=0.5 \text {, } \\
& r_{43}^{-}=1-r_{34}^{+}=0.6, r_{43}^{+}=1-r_{34}^{-}=0.7 \text {, } \\
& r_{51}^{-}=1-r_{15}^{+}=0.2, r_{51}^{+}=1-r_{15}^{-}=0.8, r_{52}^{-}=1-r_{25}^{+}=-0.1, r_{52}^{+}=1-r_{25}^{-}=0.4 \text {, } \\
& r_{53}^{-}=1-r_{35}^{+}=0.3, r_{53}^{+}=1-r_{35}^{-}=0.6, r_{54}^{-}=1-r_{45}^{+}=0.2, r_{54}^{+}=1-r_{45}^{-}=0.4 \text {, } \\
& r_{61}^{-}=1-r_{16}^{+}=0.1, r_{61}^{+}=1-r_{16}^{-}=0.9, r_{62}^{-}=1-r_{26}^{+}=-0.2, r_{62}^{+}=1-r_{26}^{-}=0.5 \text {, } \\
& r_{63}^{-}=1-r_{36}^{+}=0.2, r_{63}^{+}=1-r_{36}^{-}=0.7, r_{64}^{-}=1-r_{46}^{+}=0.1, r_{64}^{+}=1-r_{46}^{-}=0.5 \text {, } \\
& r_{65}^{-}=1-r_{56}^{+}=0.4, r_{65}^{+}=1-r_{56}^{-}=0.6 \text {, } \\
& r_{71}^{-}=1-r_{17}^{+}=0, r_{71}^{+}=1-r_{17}^{-}=0.8, r_{72}^{-}=1-r_{27}^{+}=-0.3, r_{72}^{+}=1-r_{27}^{-}=0.4 \text {, } \\
& r_{73}^{-}=1-r_{37}^{+}=0.1, r_{73}^{+}=1-r_{37}^{-}=0.6, r_{74}^{-}=1-r_{47}^{+}=0, r_{74}^{+}=1-r_{47}^{-}=0.4 \text {, } \\
& r_{75}^{-}=1-r_{57}^{+}=0.3, r_{75}^{+}=1-r_{57}^{-}=0.5, r_{76}^{-}=1-r_{67}^{+}=0.4, r_{76}^{+}=1-r_{67}^{-}=0.4 \text {. }
\end{aligned}
$$

Thus, 


$$
\dot{\bar{R}}=\left[\begin{array}{ccccccc}
{[0.5,0.5]} & {[0.1,0.2]} & {[0.3,0.6]} & {[0.1,0.5]} & {[0.2,0.8]} & {[0.1,0.9]} & {[0.2,1]} \\
{[0.8,0.9]} & {[0.5,0.5]} & {[0.7,0.9]} & {[0.5,0.8]} & {[0.6,1.1]} & {[0.5,1.2]} & {[0.6,1.3]} \\
{[0.4,0.7]} & {[0.1,0.3]} & {[0.5,0.5]} & {[0.3,0.4]} & {[0.4,0.7]} & {[0.3,0.8]} & {[0.4,0.9]} \\
{[0.5,0.9]} & {[0.2,0.5]} & {[0.6,0.7]} & {[0.5,0.5]} & {[0.6,0.8]} & {[0.5,0.9]} & {[0.6,1]} \\
{[0.2,0.8]} & {[-0.1,0.4]} & {[0.3,0.6]} & {[0.2,0.4]} & {[0.5,0.5]} & {[0.4,0.6]} & {[0.5,0.7]} \\
{[0.1,0.9]} & {[-0.2,0.5]} & {[0.2,0.7]} & {[0.1,0.5]} & {[0.4,0.6]} & {[0.5,0.5]} & {[0.6,0.6]} \\
{[0,0.8]} & {[-0.3,0.4]} & {[0.1,0.6]} & {[0,0.4]} & {[0.3,0.5]} & {[0.4,0.4]} & {[0.5,0.5]}
\end{array}\right]
$$

As the preference relation contains values falling outside the interval $[0,1]$ and $c=0.3$, a transformation function $f(x)=\frac{x+c}{1+2 c}$ is applied to the lower and upper bounds of each interval value in $\dot{\bar{R}}$, yielding

$$
\ddot{\bar{R}}=\left[\begin{array}{cccccccc}
{[0.5,0.5]} & {[0.25,0.31]} & {[0.38,0.56]} & {[0.25,0.5]} & {[0.31,0.69]} & {[0.25,0.75]} & {[0.31,0.81]} \\
{[0.69,0.75]} & {[0.5,0.5]} & {[0.63,0.75]} & {[0.5,0.69]} & {[0.56,0.88]} & {[0.5,0.94]} & {[0.56,1]} \\
{[0.44,0.63]} & {[0.25,0.38]} & {[0.5,0.5]} & {[0.38,0.44]} & {[0.44,0.63]} & {[0.38,0.69]} & {[0.44,0.75]} \\
{[0.5,0.75]} & {[0.31,0.5]} & {[0.56,0.63]} & {[0.5,0.5]} & {[0.56,0.69]} & {[0.5,0.75]} & {[0.56,0.81]} \\
{[0.31,0.69]} & {[0.13,0.44]} & {[0.38,0.56]} & {[0.31,0.44]} & {[0.5,0.5]} & {[0.44,0.56]} & {[0.5,0.63]} \\
{[0.25,0.75]} & {[0.06,0.5]} & {[0.31,0.68]} & {[0.25,0.5]} & {[0.44,0.56]} & {[0.5,0.5]} & {[0.56,0.56]} \\
{[0.19,0.69]} & {[0,0.44]} & {[0.25,0.56]} & {[0.19,0.44]} & {[0.38,0.5]} & {[0.44,0.44]} & {[0.5,0.5]}
\end{array}\right]
$$

Alonso et al. (2008) proposed a different procedure to estimate missing values in an incomplete IFRP (see Appendix). For a comparison with our approach, their procedure is applied to determine the missing judgments in this example.

By Eq. (32), $R L$ and $R R$ are obtained as follows:

$$
R L=\left[\begin{array}{ccccccc}
0.5 & x & x & x & x & x & x \\
x & 0.5 & x & x & x & x & x \\
0.4 & 0.1 & 0.5 & 0.3 & 0.4 & 0.3 & 0.4 \\
x & x & x & 0.5 & x & x & x \\
x & x & x & x & 0.5 & x & x \\
x & x & x & x & x & 0.5 & x \\
x & x & x & x & x & x & 0.5
\end{array}\right], R R=\left[\begin{array}{ccccccc}
0.5 & x & x & x & x & x & x \\
x & 0.5 & x & x & x & x & x \\
0.7 & 0.3 & 0.5 & 0.4 & 0.7 & 0.8 & 0.9 \\
x & x & x & 0.5 & x & x & x \\
x & x & x & x & 0.5 & x & x \\
x & x & x & x & x & 0.5 & x \\
x & x & x & x & x & x & 0.5
\end{array}\right]
$$

Step 1. In $R L$ and $R R$, " $x$ " denotes an unknown value, and by Eq. (35), $E V$ of the known values are determined as:

$$
E V=\{(3,1),(3,2),(3,4),(3,5),(3,6),(3,7)\}
$$

Step 2. By applying Eqs. (36)-(46), one has: 


$$
\begin{aligned}
& H_{12}^{11}=\phi, \Rightarrow c r l_{12}^{1}=0, c r r_{12}^{1}=0 \\
& H_{12}^{12}=\{3\} \\
& \Rightarrow c r l_{12}^{2}= r l_{32}-r l_{31}+0.5=0.1-0.4+0.5=0.2, c r r_{12}^{1}=r r_{32}-r r_{31}+0.5=0.3-0.7+0.5=0.1 . \\
& H_{12}^{13}=\phi, \Rightarrow c r l_{12}^{3}=0, c r r_{12}^{3}=0 \\
& \kappa=1 \quad \Rightarrow c r l_{12}=\frac{0+0.2+0}{1}=0.2, c r r_{12}=\frac{0+0.1+0}{1}=0.1 .
\end{aligned}
$$

Similarly, we can get

$$
\begin{aligned}
& \operatorname{crl}_{14}=0.4, \mathrm{crr}_{14}=0.2, \mathrm{crl}_{15}=0.5, \mathrm{crr}_{15}=0.5, \mathrm{crl}_{16}=0.4, \mathrm{crr}_{16}=0.6, \\
& \mathrm{crl}_{17}=0.5, \mathrm{crr}_{17}=0.7,
\end{aligned}
$$

After the first iteration $(h=1)$, we have

$$
\bar{R}=\left[\begin{array}{ccccccc}
{[0.5,0.5]} & {[0.2,0.1]} & x & {[0.4,0.2]} & {[0.5,0.5]} & {[0.4,0.6]} & {[0.5,0.7]} \\
x & {[0.5,0.5]} & x & x & x & x & x \\
{[0.4,0.7]} & {[0.1,0.3]} & {[0.5,0.5]} & {[0.3,0.4]} & {[0.4,0.7]} & {[0.3,0.8]} & {[0.4,0.9]} \\
x & x & x & {[0.5,0.5]} & x & x & x \\
x & x & x & x & {[0.5,0.5]} & x & x \\
x & x & x & x & x & {[0.5,0.5]} & x \\
x & x & x & x & x & x & {[0.5,0.5]}
\end{array}\right]
$$

By repeating the estimation procedure, we finally have:

$$
R L=\left[\begin{array}{ccccccc}
0.5 & 0.2 & 0.6 & 0.4 & 0.5 & 0.4 & 0.5 \\
0.8 & 0.5 & 0.9 & 0.7 & 0.8 & 0.7 & 0.8 \\
0.4 & 0.1 & 0.5 & 0.3 & 0.4 & 0.3 & 0.4 \\
0.6 & 0.3 & 0.7 & 0.5 & 0.6 & 0.5 & 0.6 \\
0.5 & 0.2 & 0.6 & 0.4 & 0.5 & 0.4 & 0.5 \\
0.6 & 0.3 & 0.7 & 0.5 & 0.6 & 0.5 & 0.6 \\
0.5 & 0.2 & 0.6 & 0.4 & 0.5 & 0.4 & 0.5
\end{array}\right], R R=\left[\begin{array}{ccccccc}
0.5 & 0.1 & 0.3 & 0.2 & 0.5 & 0.6 & 0.7 \\
0.9 & 0.5 & 0.7 & 0.6 & 0.9 & 1 & 1 \\
0.7 & 0.3 & 0.5 & 0.4 & 0.7 & 0.8 & 0.9 \\
0.8 & 0.4 & 0.6 & 0.5 & 0.8 & 0.9 & 1 \\
0.5 & 0.1 & 0.3 & 0.2 & 0.5 & 0.6 & 0.7 \\
0.4 & 0 & 0.2 & 0.1 & 0.4 & 0.5 & 0.6 \\
0.3 & 0 & 0.1 & 0 & 0.3 & 0.4 & 0.5
\end{array}\right]
$$

and 


$$
\bar{R}=\left[\begin{array}{ccccccc}
{[0.5,0.5]} & {[0.2,0.1]} & {[0.6,0.3]} & {[0.4,0.2]} & {[0.5,0.5]} & {[0.4,0.6]} & {[0.5,0.7]} \\
{[0.8,0.9]} & {[0.5,0.5]} & {[0.9,0.7]} & {[0.7,0.6]} & {[0.8,0.9]} & {[0.7,1]} & {[0.8,1]} \\
{[0.4,0.7]} & {[0.1,0.3]} & {[0.5,0.5]} & {[0.3,0.4]} & {[0.4,0.7]} & {[0.3,0.8]} & {[0.4,0.9]} \\
{[0.6,0.8]} & {[0.3,0.4]} & {[0.7,0.6]} & {[0.5,0.5]} & {[0.6,0.8]} & {[0.5,0.9]} & {[0.6,1]} \\
{[0.5,0.5]} & {[0.2,0.1]} & {[0.6,0.3]} & {[0.4,0.2]} & {[0.5,0.5]} & {[0.4,0.6]} & {[0.5,0.7]} \\
{[0.6,0.4]} & {[0.3,0]} & {[0.7,0.2]} & {[0.5,0.1]} & {[0.6,0.4]} & {[0.5,0.5]} & {[0.6,0.6]} \\
{[0.5,0.3]} & {[0.2,0]} & {[0.6,0.1]} & {[0.4,0]} & {[0.5,0.3]} & {[0.4,0.4]} & {[0.5,0.5]}
\end{array}\right]
$$

This example illustrates some drawbacks of Alonso et al. (2008)'s iterative process. Firstly, after the first iteration $(h=1)$, some estimated missing values such as $\bar{r}_{12}$ and $\bar{r}_{14}$ are undesirable because they do not constitute interval values due to $r l_{12}>r r_{12}, r l_{14}>r r_{14}$. Secondly, several iterations are needed to estimate some missing values. For example, $\bar{r}_{13}$ could not be estimated during the first iteration. Instead, it can only be estimated at a later iteration based on both judgments data provided by the DM and estimated values during earlier iterations. In contrast, our method can estimate missing values in one step and all the missing values are estimated based on the preference values furnished by the DM. Thirdly, the derived $\bar{R}$ by Alonso et al. (2008)'s method is no longer an IFPR because it does not satisfy conditions (a) and (b) of Proposition 5 or Proposition 7, while our estimated matrix $\bar{R}$ is always an additive consistent IFPR.

\subsection{An estimation procedure for acceptable incomplete IFPRs with more known judgments}

Next, we consider an acceptable incomplete IFPR $\bar{R}=\left(\bar{r}_{i j}\right)$ with more known elements (on top of the minimum $n-1$ values). In this case, by Proposition 6, each missing element $\bar{r}_{j_{k-1} j_{k}}$ in $\bar{R}=\left(\bar{r}_{i j}\right)$ can be estimated. First, find a sequence of values $r_{i_{1}}, r_{j_{1} j_{2}}, \ldots, r_{j_{i} i}\left(i<j_{1}<j_{2}<\ldots<j_{t}\right)$ that include one and only one unknown element $\bar{r}_{j_{k-1} j_{k}}$. If $j_{k-1}<j_{k}, \bar{r}_{j_{k-1} j_{k}}$ is located in the middle of the sequence, and this missing element can be estimated as $\dot{\bar{r}}_{j_{k-1} j_{k}}=\left[\dot{r}_{j_{k-1} j_{k}}^{-}, \dot{r}_{j_{k-1} j_{k}}^{+}\right]$by Eq.(23) and (24) as

$$
\begin{aligned}
& \dot{r}_{j_{k-1} j_{k}}^{-}=\frac{1}{\# \mathrm{H}} \sum_{i<j_{1}<j_{2}<\ldots<j_{t}}\left(\frac{t+1}{2}-\left(r_{i_{1}}^{-}+r_{j_{1} j_{2}}^{-}+\ldots r_{j_{k-2} j_{k-1}}^{-}+r_{j_{k} j_{k+1}}^{-} \ldots+r_{j_{t-1} j_{t}}^{-}+r_{j_{i} i}^{+}\right)\right) \\
& \dot{r}_{j_{k-1} j_{k}}^{+}=\frac{1}{\# \mathrm{H}} \sum_{i<j_{1}<j_{2}<\ldots<j_{t}}\left(\frac{t+1}{2}-\left(r_{i_{1}}^{+}+r_{j_{1} j_{2}}^{+}+\ldots r_{j_{k-2} j_{k-1}}^{+}+r_{j_{k} j_{k+1}}^{+} \ldots+r_{j_{t-1} j_{t}}^{+}+r_{j_{i} i}^{-}\right)\right), \\
& \quad \text { for all } \bar{r}_{i_{j_{1}}}, \bar{r}_{j_{1} j_{2}}, \ldots, \bar{r}_{j_{k-2} j_{k-1}}, \ldots, \bar{r}_{j_{i} i} \in \Omega
\end{aligned}
$$


If $j_{k-1}>j_{k}, \bar{r}_{j_{k-1} j_{k}}$ is the last value in the sequence, and $\bar{r}_{j_{k-1} j_{k}}$ is equal to $\bar{r}_{j_{t} i}$. In this case, it can be estimated as $\dot{\bar{r}}_{j_{k-1} j_{k}}=\left[\dot{r}_{j_{k-1} j_{k}}^{-}, \dot{j}_{j_{k-1} j_{k}}^{+}\right]$by Eq.(23) and (24), where

$$
\begin{aligned}
& \dot{r}_{j_{t} i}^{-}=\frac{1}{\# \mathrm{H}} \sum_{i<j_{1}<j_{2}<\ldots<j_{t}}\left(\frac{t+1}{2}-\left(r_{i j_{1}}^{+}+r_{j_{1} j_{2}}^{+}+\ldots+r_{j_{t-1} j_{t}}^{+}\right)\right), \\
& \dot{r}_{j_{t} i}^{+}=\frac{1}{\# \mathrm{H}} \sum_{i<j_{1}<j_{2}<\ldots<j_{t}}\left(\frac{t+1}{2}-\left(r_{i j_{1}}^{-}+r_{j_{1} j_{2}}^{-}+\ldots+r_{j_{t-1} j_{t}}^{-}\right)\right),
\end{aligned}
$$

for all $\bar{r}_{i j_{1}}, \bar{r}_{j_{1} j_{2}}, \ldots, \bar{r}_{j_{k-2} j_{k-1}} \in \Omega$

$\Omega$ is the set of all the known elements in $\bar{R}, \# \mathrm{H}$ is the number of eligible sequences $\bar{r}_{i j_{1}}, \bar{r}_{j_{1} j_{2}}, \ldots$, $\bar{r}_{j_{t} i} \cdot \bar{r}_{j_{k} j_{k-1}}$ can be automatically obtained by Eq.(22)

$$
\dot{\bar{r}}_{j_{k} j_{k-1}}=[1,1] \ominus \dot{\bar{r}}_{j_{k-1} j_{k}}, \bar{r}_{i i}=[0.5,0.5]
$$

Example 2. Consider an acceptable incomplete IFPR.

$$
\bar{R}=\left[\begin{array}{ccccc}
{[0.5,0.5]} & {[0.3,0.5]} & {[0.3,0.4]} & {[0,0.3]} & {[0.3,0.7]} \\
{[0.5,0.7]} & {[0.5,0.5]} & {[0.5,0.6]} & x & {[0.4,0.7]} \\
{[0.4,0.7]} & {[0.4,0.5]} & {[0.5,0.5]} & x & {[0.4,0.6]} \\
{[0.7,1]} & x & x & {[0.5,0.5]} & x \\
{[0.3,0.7]} & {[0.3,0.6]} & {[0.4,0.6]} & x & {[0.5,0.5]}
\end{array}\right]
$$

In order to estimate the missing value $\bar{r}_{24}$, the only sequence that contains one and only one unknown element $\bar{r}_{24}$ is $\left\{\bar{r}_{12}, \bar{r}_{24}, \bar{r}_{41}\right\}$. By Eq.(25), we have

$$
\begin{aligned}
& \dot{r}_{24}^{-}=1.5-r_{12}^{-}-r_{41}^{+}=1.5-0.3-1=0.2 \\
& \dot{r}_{24}^{+}=1.5-r_{12}^{+}-r_{41}^{-}=1.5-0.5-0.7=0.3
\end{aligned}
$$

For $\bar{r}_{34}$, there exist two eligible sequences $\left\{\bar{r}_{12}, \bar{r}_{23}, \bar{r}_{34}, \bar{r}_{41}\right\},\left\{\bar{r}_{13}, \bar{r}_{34}, \bar{r}_{41}\right\}$ that include $\bar{r}_{34}$, by Eq.(25), we have

$$
\begin{aligned}
\dot{r}_{34}^{-} & =\frac{1}{2}\left(\left(2-r_{12}^{-}-r_{23}^{-}-r_{41}^{+}\right)+\left(1.5-r_{13}^{-}-r_{41}^{+}\right)\right) \\
& =\frac{1}{2}((2-0.3-0.5-1)+(1.5-0.3-1))=0.2 \\
\dot{r}_{34}^{+} & =\frac{1}{2}\left(\left(2-r_{12}^{+}-r_{23}^{+}-r_{41}^{-}\right)+\left(1.5-r_{13}^{+}-r_{41}^{-}\right)\right)
\end{aligned}
$$




$$
=\frac{1}{2}((2-0.5-0.6-0.7)+(1.5-0.4-0.7))=0.3
$$

For $\bar{r}_{45}$, there exists a unique eligible sequence $\left\{\bar{r}_{14}, \bar{r}_{45}, \bar{r}_{51}\right\}$ containing $\bar{r}_{45}$, then we have

$$
\begin{aligned}
& \dot{r}_{45}^{-}=1.5-r_{14}^{-}-r_{51}^{+}=1.5-0-0.7=0.8 \\
& \dot{r}_{45}^{+}=1.5-r_{14}^{+}-r_{51}^{-}=1.5-0.3-0.3=0.9
\end{aligned}
$$

Thus, we have

$\dot{r}_{24}=[0.2,0.3], \dot{r}_{34}=[0.2,0.3], \dot{r}_{45}=[0.8,0.9]$

$\dot{r}_{42}=[0.7,0.8], \dot{r}_{43}=[0.7,0.8], \dot{r}_{54}=[0.1,0.2]$

Based on the aforesaid calculations, a complete additive consistent IFPR is constructed as:

$$
\dot{\bar{R}}=\left[\begin{array}{ccccc}
{[0.5,0.5]} & {[0.3,0.5]} & {[0.3,0.4]} & {[0,0.3]} & {[0.3,0.7]} \\
{[0.5,0.7]} & {[0.5,0.5]} & {[0.5,0.6]} & {[0.2,0.3]} & {[0.4,0.7]} \\
{[0.4,0.7]} & {[0.4,0.5]} & {[0.5,0.5]} & {[0.2,0.3]} & {[0.4,0.6]} \\
{[0.7,1]} & {[0.7,0.8]} & {[0.7,0.8]} & {[0.5,0.5]} & {[0.8,0.9]} \\
{[0.3,0.7]} & {[0.3,0.6]} & {[0.4,0.6]} & {[0.1,0.2]} & {[0.5,0.5]}
\end{array}\right]
$$

In the real world, many decision making processes involve multiple stakeholders rather than a single DM. Next, Algorithm II is proposed to handle group decisions with incomplete IFPRs.

\section{Algorithm II}

For a group decision making problem, let $X=\left\{x_{1}, x_{2}, \ldots, x_{n}\right\}$ be a discrete set of alternatives, $E=\left\{e_{1}, e_{2}, \ldots, e_{m}\right\}$ be a group of experts, $\lambda=\left(\lambda_{1}, \lambda_{2}, \ldots, \lambda_{m}\right)^{T}$ be the weight vector for the DMs, where $\lambda_{l} \geq 0, l=1,2, \ldots, m, \sum_{l=1}^{m} \lambda_{l}=1$. A decision procedure with acceptable incomplete IFPRs is described as follows:

Step 1. Each expert $e_{l}(l=1,2, \ldots, m)$ compares each pair of alternatives and furnishes his/her assessments as an acceptable incomplete IFPR $\bar{R}^{(l)}=\left(\bar{r}_{i j}^{(l)}\right)_{n \times n}$.

Step 2. Estimate missing elements in $\bar{R}^{(l)}=\left(\bar{r}_{i j}^{(l)}\right)_{n \times n}(l=1,2, \ldots, m)$ using the known elements through Eq.(25), and obtain complete IFPRs $\dot{\bar{R}}^{(l)}=\left(\dot{\bar{r}}_{i j}^{(l)}\right)_{n \times n}$. If any resulting preference relation contains values falling outside the interval $[0,1]$, and within the interval $[-c, 1+c]$, a 
transformation function $f(x)=\frac{x+c}{1+2 c}$ is applied to the upper and lower bounds for the interval values to convert the preference relation to an IFPR.

Step 3. Use the interval fuzzy weighted averaging operator Eq.(21) to aggregate all individual complete IFPRs $\dot{\bar{R}}^{(l)}=\left(\dot{\bar{r}}_{i j}^{(l)}\right)_{n \times n}(l=1,2, \ldots, m)$ into a collective IFPR $\dot{\bar{R}}=\left(\dot{\bar{r}}_{i j}\right)_{n \times n}$.

Step 4. Utilize the interval normalizing rank aggregation method

$$
\bar{w}_{i}=\frac{\sum_{j=1}^{n} \dot{\bar{r}}_{i j}}{\sum_{i=1}^{n} \sum_{j=1}^{n} \dot{\bar{r}_{i j}}}, i=1,2, \ldots, n
$$

to derive average degree $\bar{w}_{i}$ of the $i^{\text {th }}$ alternative over all the other alternatives.

Step 5. Utilize the following formula (Facchinetti et al., 1998; Wang et al., 2005; Xu and Da, 2002)

$$
p\left(\bar{w}_{i} \geq \bar{w}_{j}\right)=\min \left\{\max \left(\frac{w_{i}^{+}-w_{j}^{-}}{w_{i}^{+}-w_{i}^{-}+w_{j}^{+}-w_{j}^{-}}, 0\right), 1\right\}
$$

to obtain the possibility degree $p_{i j}=p\left(\bar{w}_{i} \geq \bar{w}_{j}\right)$, and construct a complementary matrix $P=\left(p_{i j}\right)_{n \times n}$, where

$$
p_{i j} \geq 0, p_{i j}+p_{j i}=1, p_{i i}=0.5, i, j=1,2, \ldots, n
$$

Step 6. Utilize the normalizing rank aggregation method (Xu et al., 2009)

$$
\varpi_{i}=\frac{\sum_{j=1}^{n} p_{i j}}{n^{2} / 2}, i=1,2, \ldots, n
$$

to derive a priority vector $\varpi=\left(\varpi_{1}, \varpi_{2}, \ldots, \varpi_{n}\right)^{T}$ based on the complementary matrix $P$. The alternatives $x_{i}(i=1,2, \ldots, n)$ are ranked in a descending order as per the values of $\varpi_{i}$ $(i=1,2, \ldots, n)$.

Step 7. End.

\section{Case study}

This section presents a group decision making problem that is concerned with evaluating and selecting potential suppliers for the Pars Solar Company (adapted from (Hadi-Vencheh and Mirjaberi, 2011)). The firm produces solar boiler and solar water refiners. In its production process, the company needs to purchase solar panels in different sizes and voltages from different suppliers. Currently, Pars Solar Company has five potential suppliers from five different countries, the U.S., Germany, China, Turkey, and Iran, denoted as $x_{i}(i=1,2, \ldots, 5)$, respectively. 
A committee consisting of three managers $e_{l}(l=1,2,3)$ (whose weighting vector is $\lambda=(1 / 3$, $1 / 3,1 / 3)^{T}$ ) from different departments has been set up to provide their assessment on the five suppliers $x_{i}(i=1,2, \ldots, 5)$. Assume that the managers $e_{l}(l=1,2,3)$ give their evaluations as the following acceptable incomplete IFPRs $\bar{R}^{(l)}=\left(\bar{r}_{i j}^{(l)}\right)_{n \times n}(l=1,2,3)$ :

$$
\begin{aligned}
\bar{R}^{(1)}= & {\left[\begin{array}{ccccc}
{[0.5,0.5]} & x & x & {[0.2,0.6]} & {[0.4,0.7]} \\
x & {[0.5,0.5]} & {[0.7,0.8]} & {[0.9,1]} & x \\
x & {[0.2,0.3]} & {[0.5,0.5]} & {[0.4,0.6]} & x \\
{[0.4,0.8]} & {[0,0.1]} & {[0.4,0.6]} & {[0.5,0.5]} & {[0.4,0.5]} \\
{[0.3,0.6]} & x & x & {[0.5,0.6]} & {[0.5,0.5]}
\end{array}\right], } \\
\bar{R}^{(2)}= & {\left[\begin{array}{ccccc}
{[0.5,0.5]} & x & {[0.2,0.5]} & x & x \\
x & {[0.5,0.5]} & {[0.3,0.5]} & x & x \\
{[0.5,0.8]} & {[0.5,0.7]} & {[0.5,0.5]} & {[0.4,0.6]} & {[0.3,0.4]} \\
x & x & {[0.4,0.6]} & {[0.5,0.5]} & x \\
x & x & {[0.6,0.8]} & x & {[0.5,0.5]}
\end{array}\right], } \\
\bar{R}^{(3)}= & {\left[\begin{array}{ccccc}
{[0.5,0.5]} & {[0.3,0.4]} & {[0.4,0.6]} & {[0.1,0.2]} & x \\
{[0.6,0.7]} & {[0.5,0.5]} & x & {[0.4,0.5]} & {[0.4,0.7]} \\
{[0.4,0.6]} & x & {[0.5,0.5]} & {[0.2,0.3]} & x \\
{[0.8,0.9]} & {[0.5,0.6]} & {[0.7,0.8]} & {[0.5,0.5]} & {[0.7,0.8]} \\
x & {[0.3,0.6]} & x & {[0.2,0.3]} & {[0.5,0.5]}
\end{array}\right] . }
\end{aligned}
$$

To illustrate the solution process of Algorithm II, the missing elements are first estimated for $\bar{R}^{(l)}=\left(\bar{r}_{i j}^{(l)}\right)_{n \times n}(l=1,2,3)$

Step 1. Using the known elements and Eq.(23), (24), we have:

$$
\begin{aligned}
\dot{r}_{12}^{(1)-} & =\frac{1}{3}\left(\left(2.5-r_{23}^{(1)-}-r_{34}^{(1)-}-r_{45}^{(1)-}-r_{51}^{(1)+}\right)+\left(2-r_{24}^{(1)-}-r_{45}^{(1)-}-r_{51}^{(1)+}\right)+\left(1.5-r_{24}^{(1)-}-r_{41}^{(1)+}\right)\right) \\
& =\frac{1}{3}((2.5-0.7-0.4-0.4-0.6)+(2-0.9-0.4-0.6)+(1.5-0.9-0.8)) \\
& =0.1 \\
\dot{r}_{12}^{(1)+} & =\frac{1}{3}\left(\left(2.5-r_{23}^{(1)+}-r_{34}^{(1)+}-r_{45}^{(1)+}-r_{51}^{(1)-}\right)+\left(2-r_{24}^{(1)+}-r_{45}^{(1)+}-r_{51}^{(1)-}\right)+\left(1.5-r_{24}^{(1)+}-r_{41}^{(1)-}\right)\right) \\
& =\frac{1}{3}((2.5-0.8-0.6-0.5-0.3)+(2-1-0.5-0.3)+(1.5-1-0.4)) \\
& =0.2
\end{aligned}
$$




$$
\begin{aligned}
\dot{r}_{13}^{(1)-}= & \frac{1}{2}\left(\left(2-r_{34}^{(1)-}-r_{45}^{(1)-}-r_{51}^{(1)+}\right)+\left(1.5-r_{34}^{(1)-}-r_{41}^{(1)+}\right)\right) \\
= & \frac{1}{2}((2-0.4-0.4-0.6)+(1.5-0.4-0.8)) \\
& =0.225 \\
\dot{r}_{13}^{(1)+}= & \frac{1}{2}\left(\left(2-r_{34}^{(1)+}-r_{45}^{(1)+}-r_{51}^{(1)-}\right)+\left(1.5-r_{34}^{(1)+}-r_{41}^{(1)-}\right)\right) \\
= & \frac{1}{2}((2-0.6-0.5-0.3)+(1.5-0.6-0.4)) \\
= & 0.55 \\
\dot{r}_{52}^{(1)-}= & \frac{1}{2}\left(\left(2-r_{23}^{(1)+}-r_{34}^{(1)+}-r_{45}^{(1)+}\right)+\left(1.5-r_{24}^{(1)+}-r_{45}^{(1)+}\right)\right) \\
= & \frac{1}{2}((2-0.8-0.6-0.5)+(1.5-1-0.5)) \\
\dot{r}_{53}^{(1)+}= & 1.5-r_{34}^{(1)-}-r_{45}^{(1)-}=1.5-0.4-0.4 \\
= & 0.7 \\
= & 0.05 \\
\dot{r}_{52}^{(1)+}= & \frac{1}{2}\left(\left(2-r_{23}^{(1)-}-r_{34}^{(1)-}-r_{45}^{(1)-}\right)+\left(1.5-r_{24}^{(1)-}-r_{45}^{(1)-}\right)\right) \\
= & \frac{1}{2}((2-0.7-0.4-0.4)+(1.5-0.9-0.4)) \\
& 0.4 \\
(1)+ & r_{45}^{(1)+}=1.5-0.6-0.5 \\
&
\end{aligned}
$$

Then we have

$\dot{r}_{12}^{(1)}=[0.1,0.2], \dot{r}_{21}^{(1)}=[0.8,0.9], \dot{r}_{13}^{(1)}=[0.225,0.55], \dot{r}_{31}^{(1)}=[0.45,0.775]$,

$\dot{r}_{52}^{(1)}=[0.05,0.35], \dot{r}_{25}^{(1)}=[0.65,0.95], \dot{r}_{53}^{(1)}=[0.4,0.7], \dot{r}_{35}^{(1)}=[0.3,0.6]$.

Based on $\bar{R}^{(1)}$ and the aforesaid calculations, a complete IFPR $\dot{\bar{R}}^{(1)}$ is constructed for DM $e_{1}$ as follows: 


$$
\dot{\bar{R}}^{(1)}=\left[\begin{array}{ccccc}
{[0.5,0.5]} & {[0.1,0.2]} & {[0.225,0.55]} & {[0.2,0.6]} & {[0.4,0.7]} \\
{[0.8,0.9]} & {[0.5,0.5]} & {[0.7,0.8]} & {[0.9,1]} & {[0.65,0.95]} \\
{[0.45,0.775]} & {[0.2,0.3]} & {[0.5,0.5]} & {[0.4,0.6]} & {[0.3,0.6]} \\
{[0.4,0.8]} & {[0,0.1]} & {[0.4,0.6]} & {[0.5,0.5]} & {[0.4,0.5]} \\
{[0.3,0.6]} & {[0.05,0.35]} & {[0.4,0.7]} & {[0.5,0.6]} & {[0.5,0.5]}
\end{array}\right]
$$

Similarly, we obtain

$$
\dot{\bar{R}}^{(2)}=\left[\begin{array}{ccccc}
{[0.5,0.5]} & {[0.4,0.5]} & {[0.2,0.5]} & {[0.1,0.6]} & {[-0.1,0.4]} \\
{[0.5,0.6]} & {[0.5,0.5]} & {[0.3,0.5]} & {[0.2,0.6]} & {[0,0.4]} \\
{[0.5,0.8]} & {[0.5,0.7]} & {[0.5,0.5]} & {[0.4,0.6]} & {[0.2,0.4]} \\
{[0.4,0.9]} & {[0.4,0.8]} & {[0.4,0.6]} & {[0.5,0.5]} & {[0.3,0.3]} \\
{[0.6,1.1]} & {[0.6,1]} & {[0.6,0.8]} & {[0.7,0.7]} & {[0.5,0.5]}
\end{array}\right]
$$

As this preference relation contains values that fall outside the interval $[0,1]$, it is necessary to apply the transformation function $f(x)=\frac{x+c}{1+2 c}$ to convert it to an IFPR as

$$
\ddot{\bar{R}}^{(2)}=\left[\begin{array}{ccccc}
{[0.5,0.5]} & {[0.42,0.5]} & {[0.25,0.5]} & {[0.17,0.58]} & {[0,0.42]} \\
{[0.5,0.58]} & {[0.5,0.5]} & {[0.33,0.5]} & {[0.25,0.58]} & {[0.08,0.42]} \\
{[0.5,0.75]} & {[0.5,0.67]} & {[0.5,0.5]} & {[0.42,0.58]} & {[0.25,0.42]} \\
{[0.42,0.83]} & {[0.42,0.75]} & {[0.42,0.58]} & {[0.5,0.5]} & {[0.33,0.33]} \\
{[0.58,1]} & {[0.58,0.92]} & {[0.58,0.75]} & {[0.67,0.67]} & {[0.5,0.5]}
\end{array}\right]
$$

For DM $e_{3}$, one can easily obtain its complete IFPR as

$$
\dot{\bar{R}}^{(3)}=\left[\begin{array}{ccccc}
{[0.5,0.5]} & {[0.3,0.4]} & {[0.4,0.6]} & {[0.1,0.2]} & {[0.333,0.633]} \\
{[0.6,0.7]} & {[0.5,0.5]} & {[0.6,0.625]} & {[0.4,0.5]} & {[0.4,0.7]} \\
{[0.4,0.6]} & {[0.375,0.4]} & {[0.5,0.5]} & {[0.2,0.3]} & {[0.4,0.6]} \\
{[0.8,0.9]} & {[0.5,0.6]} & {[0.7,0.8]} & {[0.5,0.5]} & {[0.7,0.8]} \\
{[0.367,0.667]} & {[0.3,0.6]} & {[0.4,0.6]} & {[0.2,0.3]} & {[0.5,0.5]}
\end{array}\right]
$$

Step 2. Utilize the interval additive weighted averaging operator Eq.(21) to fuse the constructed complete IFRPs $\dot{\bar{R}}^{(1)}, \ddot{\bar{R}}^{(2)}, \dot{\bar{R}}^{(3)}$ into a collective complete IFPR $\dot{\bar{R}}=\left(\dot{\overline{\bar{r}_{i j}}}\right)_{n \times n}$ as

$$
\dot{\bar{R}}=\left[\begin{array}{ccccc}
{[0.5,0.5]} & {[0.273,0.367]} & {[0.292,0.55]} & {[0.157,0.46]} & {[0.244,0.584]} \\
{[0.633,0.727]} & {[0.5,0.5]} & {[0.543,0.642]} & {[0.517,0.693]} & {[0.377,0.69]} \\
{[0.45,0.708]} & {[0.358,0.457]} & {[0.5,0.5]} & {[0.34,0.493]} & {[0.317,0.54]} \\
{[0.54,0.843]} & {[0.307,0.483]} & {[0.507,0.66]} & {[0.5,0.5]} & {[0.477,0.543]} \\
{[0.416,0.756]} & {[0.31,0.623]} & {[0.46,0.683]} & {[0.457,0.523]} & {[0.5,0.5]}
\end{array}\right]
$$


Step 3. By employing the interval normalizing rank aggregation method Eq.(31), one can derive the average degree $\bar{w}_{i}$ for each alternative as

$$
\begin{aligned}
& \bar{w}_{1}=[0.1009,0.2350], \bar{w}_{2}=[0.1769,0.3105], \bar{w}_{3}=[0.1353,0.2576], \bar{w}_{4}=[0.1604,0.2893], \\
& \bar{w}_{5}=[0.1475,0.2946] .
\end{aligned}
$$

Step 4. By using Eq.(29) to compare each $\bar{w}_{i}$ with each $\bar{w}_{j}(j=1,2, \ldots, n)$, we develop a complementary matrix $P=\left(p_{i j}\right)_{n \times n}$ as

$$
P=\left[\begin{array}{ccccc}
0.5 & 0.217 & 0.389 & 0.284 & 0.311 \\
0.783 & 0.5 & 0.685 & 0.572 & 0.581 \\
0.611 & 0.315 & 0.5 & 0.387 & 0.409 \\
0.716 & 0.428 & 0.613 & 0.5 & 0.514 \\
0.689 & 0.419 & 0.591 & 0.486 & 0.5
\end{array}\right]
$$

Step 5. By applying the normalizing rank aggregation method (Xu et al., 2009), one has

$$
\varpi=(0.136,0.250,0.178,0.222,0.215)^{T}
$$

A final ranking of the alternatives can thus be derived as follows:

$$
x_{2} \succ x_{5} \succ x_{4} \succ x_{3} \succ x_{1}
$$

Therefore, the best alternative is $x_{2}$.

We can also compare the final result with what is implied in the initial incomplete input. From $\bar{R}^{(1)}$, it can be determined that the partial ranking order by the first manager $e_{1}$ is $x_{2} \succ x_{3}, x_{2} \succ x_{4}$, the partial ranking order by the third manager $e_{3}$ is $x_{2} \succ x_{1}, x_{4} \succ x_{5}$. Based on these partial orderings, it is sensible to expect that $x_{2}$ arise as the best alternative, consistent with the prediction by our model here.

Numerical examples illustrate how to apply the proposed method to construct complete IFPRs based on acceptable incomplete IFPRs. Generally speaking, the proposed approach is relatively easy for use in determining missing values. More importantly, Herrera-Viedma et al. (2004) proposed a method to construct a consistent fuzzy preference relation from $n-1$ preference values $\left\{r_{12}, r_{23}, \ldots, r_{n-1 n}\right\}$. Sometimes, this may be a challenge for the DM to provide his/her consecutive pairwise judgments $\left\{r_{12}, r_{23}, \ldots, r_{n-1 n}\right\}$. Based on Proposition 10, if $\bar{R}$ is an acceptable incomplete 
IFPR, then there exists at least one known non-diagonal element in each row or each column of $\bar{R}$. Therefore, an acceptable incomplete IFPR with at least (n-1) judgments provided by the DM. $\left\{r_{12}, r_{23}, \ldots, r_{n-1 n}\right\}$ is only one example that makes up an acceptable incomplete IFPR. The proposed approach herein is able to handle any acceptable incomplete IFPR with $n-1$ judgment data such as $\left\{r_{12}, r_{13}, \ldots, r_{1 n}\right\},\left\{r_{21}, r_{23}, \ldots, r_{2 n}\right\},\left\{r_{21}, r_{31}, \ldots, r_{n 1}\right\}$. If the DM can provide more judgments, one can use the sequence(s) of known values $r_{i j_{1}}, r_{j_{1} j_{2}}, \ldots, r_{j_{t-1} j_{t}}, r_{j_{t} i}$ to estimate the missing values. Therefore, our proposed approach can be applied to more general IFPRs.

\section{Conclusions}

This paper first extends an additive consistency property (Herrera-Viedma et al., 2004) of fuzzy preference relations to a more general case. This property is then generalized to IFPRs based on additive transitivity. After further characterizing additive consistent IFPRs, we propose a method to construct additive consistent IFPRs from acceptable incomplete IFPRs. Numerical studies illustrate that the proposed method can handle acceptable incomplete IFPRs with as few as $n-1$ judgment data. Subsequently, another algorithm is developed to deal with GDM problems with acceptable incomplete IFPRs. This procedure is composed of two phases, the estimation of missing preference values and the selection of the best alternative(s) where missing elements are determined by the sequences of known values $r_{i j_{1}}, r_{j_{1} j_{2}}, \ldots, r_{j_{t-1} j_{t}}, r_{j_{t}}$.

In the future, the proposed framework will be applied to other group decision making problems such as supplier selection (Chen et al., 2006), e-business (Mohanty and Bhasker, 2005), technology adoption (Choudhury et al., 2006), broadband internet service selection (Wang and Parkan, 2008), and air-conditioning systems selection (Xu, 2009).

\section{Acknowledgement}

The authors would like to thank the two anonymous referees for their insightful and constructive comments and suggestions, which have led to this improved version of the paper.

\section{Appendix}

Alonso et al. (2008) proposed the following procedure to estimate missing values for IFPRs. 
An IFPR $\bar{R}=\left(\bar{r}_{i j}\right)$ can be viewed as two "independent" fuzzy preference relations, the first one $R L$ corresponding to the left bounds of the intervals and the second one $R R$ corresponding to the right bounds of the intervals.

$$
\bar{R}=\left(\bar{r}_{i j}\right)=\left(\left[r l_{i j}, r r_{i j}\right]\right) \text { with } R L=\left(r l_{i j}\right) \text { and } R R=\left(r r_{i j}\right) \text { and } r l_{i j} \leq r r_{i j} \forall i, j
$$

Step 1. Determine the set $B$, the set $M V$ and the set $E V$ as follows:

$$
\begin{aligned}
& B=\{(i, j) \mid i, j \in\{1, \ldots, n\} \wedge i \neq j\} \\
& M V=\left\{(i, j) \in B \mid r_{i j} \text { is unknown }\right\} \\
& E V=B \backslash M V
\end{aligned}
$$

where $M V$ is the set of pairs of alternatives for which the preference degree of the first alternative over the second one is unknown or missing; $E V$ is the set of pairs of alternatives for which the expert provides preference values, and the symbol “"” denotes the exclusion relation. In Alonso et al.(2008)'s method, it does not take into account the preference value of one alternative over itself as $x_{i} \sim x_{i}$ is always assumed.

Step 2. If $M V=\phi$, then go to Step 4. Otherwise, the following iterative procedure is used to estimate the missing value $c r l_{i k}$ and $c r r_{i k}$ in the set $E M V_{h}$ at the $h^{\text {th }}$ iteration, where $E M V_{0}=\phi$, $(i, k) \in E M V_{h}$, and $h \geq 1$, shown as follows:

$$
\begin{aligned}
& H_{i k}^{h 1}=\left\{j \neq i, k \mid(i, j),(j, k) \in\left\{E V \bigcup_{l=0}^{h-1} E M V_{l}\right\}\right\} ; \\
& H_{i k}^{h 2}=\left\{j \neq i, k \mid(j, k),(j, i) \in\left\{E V \bigcup_{l=0}^{h-1} E M V_{l}\right\}\right\} \text {; } \\
& H_{i k}^{h 3}=\left\{j \neq i, k \mid(i, j),(k, j) \in\left\{E V \bigcup_{l=0}^{h-1} E M V_{l}\right\}\right\} \text {; } \\
& E M V_{h}=\left\{(i, k) \in M V \backslash \bigcup_{l=0}^{h-1} E M V_{l} \mid i \neq k \text { and } \exists j \in\left\{H_{i k}^{h 1} \cup H_{i k}^{h 2} \cup H_{i k}^{h 3}\right\}\right\} \\
& c r l_{i k}^{1}=\left\{\begin{array}{l}
\frac{1}{\# H_{i k}^{h 1}} \sum_{j \in H_{i k}^{h 1}} c r l_{i k}^{j 1}, \text { if } \# H_{i k}^{h 1} \neq 0 \\
0, \text { otherwise }
\end{array}, c r r_{i k}^{1}=\left\{\begin{array}{l}
\frac{1}{\# H_{i k}^{h 1}} \sum_{j \in H_{i k}^{h 1}} c r r_{i k}^{j 1}, \text { if } \# H_{i k}^{h 1} \neq 0 \\
0, \text { otherwise }
\end{array}\right.\right.
\end{aligned}
$$




$$
\begin{aligned}
& c r l_{i k}^{2}=\left\{\begin{array}{l}
\frac{1}{\# H_{i k}^{h 2}} \sum_{j \in H_{i k}^{h 1}} c r l_{i k}^{j 2}, \text { if } \# H_{i k}^{h 2} \neq 0 \\
0, \text { otherwise }
\end{array}, c r r_{i k}^{2}=\left\{\begin{array}{l}
\frac{1}{\# H_{i k}^{h 2}} \sum_{j \in H_{i k}^{h 1}} c r r_{i k}^{j 2}, \text { if } \# H_{i k}^{h 2} \neq 0 \\
0, \text { otherwise }
\end{array}\right.\right. \\
& c r l_{i k}^{3}=\left\{\begin{array}{l}
\frac{1}{\# H_{i k}^{h 3}} \sum_{j \in H_{i k}^{h 1}} c r l_{i k}^{j 3}, \text { if } \# H_{i k}^{h 3} \neq 0 \\
0, \text { otherwise }
\end{array}, \quad c r r_{i k}^{3}=\left\{\begin{array}{l}
\frac{1}{\# H_{i k}^{h 3}} \sum_{j \in H_{i k}^{h 1}} c r r_{i k}^{j 3}, \text { if } \# H_{i k}^{h 3} \neq 0 \\
0, \text { otherwise }
\end{array}\right.\right. \\
& c r l_{i k}=\frac{c r l_{i k}^{1}+c r l_{i k}^{2}+c r l_{i k}^{3}}{\kappa}, c r r_{i k}=\frac{c r r_{i k}^{1}+c r r_{i k}^{2}+c r r_{i k}^{3}}{\kappa} \\
& \kappa=\left\{\begin{array}{l}
\# H_{i k}^{h 1}+\# H_{i k}^{h 2}+\# H_{i k}^{h 3}, \text { if } \# H_{i k}^{h 1}+\# H_{i k}^{h 2}+\# H_{i k}^{h 3} \neq 0 \\
1, \text { otherwise }
\end{array}\right.
\end{aligned}
$$

where the symbol "\#” denotes the number of elements in a given set,

$$
c r l_{i k}^{j 1}=r l_{i j}+r l_{j k}-0.5, c r l_{i k}^{j 2}=r l_{j k}-r l_{j i}+0.5, c r l_{i k}^{j 3}=r l_{i j}-r l_{k j}+0.5,
$$

and

$$
c r r_{i k}^{j 1}=r r_{i j}+r r_{j k}-0.5, c r r_{i k}^{j 2}=r r_{j k}-r r_{j i}+0.5, c r r_{i k}^{j 3}=r r_{i j}-r r_{k j}+0.5,
$$

Step 3. if $\mathrm{crl}_{i k}<0$ or $\mathrm{Crr}_{i k}<0$, then $\mathrm{crl}_{i k}=0$ or $\mathrm{crr}_{i k}=0$

$$
\text { else if } \mathrm{crl}_{i \mathrm{k}}>1 \text { or } \mathrm{crr}_{i k}>1 \text {, then } \mathrm{Crl}_{i k}=1 \text { or } \mathrm{crr}_{i k}=1
$$

Step 4. End.

\section{References}

Alonso, S., Chiclana, F., Herrera, F., Herrera-Viedma, E., 2004. A learning procedure to estimate missing values in fuzzy preference relations based on additive consistency. In: MDAI2004,LNAI 3131, V. Torra, Y. Narukawa. Springer-Verlag Berlin Heidelberg, pp. 227-238.

Alonso, S., Chiclana, F., Herrera, F., Herrera-Viedma, E., Alcalá-Fdez, J., Porcel, C., 2008. A consistencybased procedure to estimate missing pairwise preference values. International Journal of Intelligent Systems, 23, 155-175.

Arbel, A., 1989. Approximate articulation of preference priority derivation. European Journal of Operational Research, 43, 317-326.

Chen, C.T., Lin, C.T., Huang, S.F., 2006. A fuzzy approach for supplier evaluation and selection in supply chain management. International Journal of Production Economics, 102, 289-301.

Chiclana, F., Herrera-Viedma, E., Alonso, S., 2009. A note on two methods for estimating missing pairwise preference values. IEEE Transactions on Systems Man and Cybernetics Part BCybernetics, 39, 1628-1633.

Chiclana, F., Herrera-Viedma, E., Alonso, S., Herrera, F., 2008. A note on the estimation of missing pairwise preference values: A U-consistency based method. International Journal of Uncertainty, Fuzziness and Knowledge-Based Sysetems, 16, 19-32. 
Chiclana, F., Herrera, F., Herrera-Viedma, E., 2002. A note on the internal consistency of various preference representations. Fuzzy Sets and Systems, 131, 75-78.

Choudhury, A.K., Shankar, R., Tiwari, M.K., 2006. Consensus-based intelligent group decision-making model for the selection of advanced technology. Decision Support Systems, 42, 1776-1799.

Csutora, R., Buckley, J.J., 2001. Fuzzy hierarchical analysis: the Lambda-Max method. Fuzzy Sets and Systems, 120, 181-195.

Facchinetti, G., Ricci, R.G., Muzzioli, S., 1998. Note on ranking fuzzy triangular numbers. International Journal of Intelligent Systems, 13, 613-622.

Fedrizzi, M., Silvio, G., 2007. Incomplete pairwise comparison and consistency optimization. European Journal of Operational Research, 183, 303-313.

Genç, S., Boran, F.E., Akay, D., Xu, Z.S., 2010. Interval multiplicative transitivity for consistency, missing values and priority weights of interval fuzzy preference relations. Information Sciences, 180, 48774891.

Hadi-Vencheh, A., Mirjaberi, M., 2011. Seclusion-factor method to solve fuzzy-multiple criteria decisiomaking problems. IEEE Transactions on Fuzzy Systems, 19, 201-209.

Hayes, B., 2003. A lucid interval. American Science, 91, 484-488.

Herrera-Viedma, E., Alonso, S., Chiclana, F., Herrera, F., 2007a. A consensus model for group decision making with incomplete fuzzy preference relations. IEEE Transactions on Fuzzy Systems, 15, 863877.

Herrera-Viedma, E., Chiclana, F., Herrera, F., Alonso, S., 2007b. Group decision-making model with incomplete fuzzy preference relations based on additive consistency. IEEE Transactions on Systems, Man, and Cybernetics-Part B:Cybernetics., 37, 176-189.

Herrera-Viedma, E., Herrera, F., Chiclana, F., Luque, M., 2004. Some issues on consistency of fuzzy preference relations. European Journal of Operational Research, 154, 98-109.

Herrera, F., Martíze, L., Sánchez, P.J., 2005. Managing non-homogeneous information in group decision making. European Journal of Operational Research, 166, 115-132.

Islam, R., Biswal, M.P., Alam, S.S., 1997. Preference programming and inconsistent interval judgments. European Journal of Operational Research, 97, 53-62.

Jiang, Y.L., 2007. An approach to group decision making based on interval fuzzy relations. Journal of Systems Science and Systems Engineering, 16, 113-120.

Kacprzyk, J., 1986. Group decision making with a fuzzy majority. Fuzzy Sets and Systems, 18, 105-118.

Kress, M., 1991. Approximate articulation of preference and priority derivation-a comment. European Journal of Operational Research, 52, 382-383.

Lee, H.S., Chou, M.T., Fang, H.H., Tseng, W.K., Yeh, C.H., 2007. Estimating missing values in incomplete additive fuzzy preference relations. In: KES 2007/ WIRN 2007, LNAI. 4693, B. Apolloni, ed., pp. 1307-1314.

Liu, F., Zhang, W.G., Wang, Z.X., 2012a. A goal programming model for incomplete interval multiplicative preference relations and its application in group decision-making. European Journal of Operational Research, 218, 747-754.

Liu, X.W., Pan, Y.W., Xu, Y.J., Yu, S., 2012b. Least square completion and inconsistency repair methods for additively consistent fuzzy preference relations. Fuzzy Sets and Systems, 198, 1-19.

Mikhailov, L., 2002. Fuzzy analytical approach to partnership selection in formation of virtual enterprises. Omega, 30, 393-401.

Mikhailov, L., 2004. A fuzzy approach to deriving priorities from interval pairwise comparison judgements. European Journal of Operational Research, 159, 687-704.

Mohanty, B.K., Bhasker, B., 2005. Product classification in the Internet business - a fuzzy approach. Decision Support Systems, 38, 611-619.

Moore, R.E., 1966. Interval Analysis, Vol., Prentice-Hall, Englewood Cliffs. 
Orlovsky, S.A., 1978. Decision-making with a fuzzy preference relation. Fuzzy Sets and Systems, 1, 155167.

Saaty, T.L., 1980. The Analytic Hierarchy Process, Vol., McGraw-Hill, New York.

Saaty, T.L., Vargas, L.G., 1987. Uncertainty and rank order in the analytic hierarchy process. European Journal of Operational Research, 32, 107-117.

Tanino, T., 1984. Fuzzy preference orderings in group decision making. Fuzzy Sets and Systems, 12, 117131.

Wang, T.C., Chen, Y.H., 2007. Applying consistent fuzzy preference relations to partnership selection. Omega, 35, 384-388.

Wang, T.C., Chen, Y.H., 2008. Applying fuzzy linguistic preference relations to the improvement of consistency of fuzzy AHP. Information Sciences, 178, 3755-3765.

Wang, Y.M., 2006. On lexicographic goal programming method for obtaining interval weights from an interval comparison matrix. Applied Mathematics and Computation, 173, 985-991.

Wang, Y.M., Chin, K.S., 2006. An eigenvector method for generating normalized interval and fuzzy weights. Applied Mathematics and Computation, 181, 1257-1275.

Wang, Y.M., Elhag, T.M.S., 2007. A goal programming method for obtaining interval weights from an interval comparison matrix. European Journal of Operational Research, 177, 458-471.

Wang, Y.M., Fan, Z.P., 2007. Fuzzy preference relations: aggregationand weight determination. Computers \& Industrial Engineering, 53, 163-172.

Wang, Y.M., Parkan, C., 2008. Optimal aggregation of fuzzy preference relations with an application to broadband internet service selection. European Journal of Operational Research, 187, 1476-1486.

Wang, Y.M., Yang, J.B., Xu, D.L., 2005. A two-stage logarithmic goal programming method for generating weights from interval comparison matrices. Fuzzy Sets and Systems, 152, 475-498.

Wang, Z.J., Li, K.W., 2012. Goal programming approaches to deriving interval weights based on interval fuzzy preference relations. Information Sciences, 193, 180-198.

Xia, M.M., Xu, Z.S., Wang, Z., 2012. Multiplicative consistency-based decision support system for incomplete linguistic preference relations. International Journal of Systems Science, http://dx.doi.org/10.1080/00207721.2012.724112.

$\mathrm{Xu}$, Y.J., 2010. Note on "The induced continuous ordered weighted geometric operators and their application in group decision making". Computers \& Industrial Engineering, 59, 365-366.

Xu, Y.J., Da, Q.L., 2008. Weighted least-square method and its improvment for priority of incomplete complementary judgement matrix. Systems Engineering and Electronics, 30, 1273-276.

Xu, Y.J., Da, Q.L., 2009. Methods for priority of incomplete complementary judgement matrices. Systems Engineering and Electronics, 31, 95-99.

Xu, Y.J., Da, Q.L., Liu, L.H., 2009. Normalizing rank aggregation method for priority of a fuzzy preference relation and its effectiveness. International Journal of Approximate Reasoning, 50, 1287-1297.

Xu, Y.J., Da, Q.L., Wang, H.M., 2010. A note on group decision-making procedure based on incomplete reciprocal relations. Soft Computing, 15, 1289-1300.

Xu, Y.J., Gupta, J.N.D., Wang, H.M., 2013a. The ordinal consistency of an incomplete reciprocal preference relation. Fuzzy Sets and Systems, http://dx.doi.org/10.1016/j.fss.2013.08.001.

Xu, Y.J., Patnayakuni, R., Wang, H.M., 2013b. Logarithmic least squares method to priority for group decision making with incomplete fuzzy preference relations. Applied Mathematical Modelling, 37, 2139-2152.

Xu, Y.J., Patnayakuni, R., Wang, H.M., 2013c. The ordinal consistency of a fuzzy preference relation. Information Sciences, 224, 152-164.

$\mathrm{Xu}$, Z.S., 2004a. Goal programming models for obtaining the priority vector of incomplete fuzzy preference relation. International Journal of Approximate Reasoning, 36, 261-270. 
Xu, Z.S., 2004b. On compatibility of interval fuzzy preference relations. Fuzzy Optimization and Decision Making, 3, 217-225.

$\mathrm{Xu}, \mathrm{Z} . \mathrm{S} ., 2005$. A procedure for decision making based on incomplete fuzzy preference relation. Fuzzy Optimization and Decision Making, 4, 175-189.

$\mathrm{Xu}, \mathrm{Z} . \mathrm{S} ., 2$ 2009. An automatic approach to reaching consensus in multiple attribute group decision making. Computers \& Industrial Engineering, 56, 1369-1374.

$\mathrm{Xu}$, Z.S., 2012. A consensus reaching process under incomplete multiplicative preference relations. International Journal of General Systems, 41, 333-351.

$\mathrm{Xu}$, Z.S., Chen, J., 2008a. Group decision-making procedure based on incomplete reciprocal relations. Soft Computing, 12, 515-521.

Xu, Z.S., Chen, J., 2008b. Some methods for deriving the priority weights from interval fuzzy preference relations. European Journal of Operational Research, 184, 266-280.

Xu, Z.S., Da, Q.L., 2002. The uncertain OWA operator. International Journal of Intelligent Systems, 17, 569-575. 